University of Rhode Island

DigitalCommons@URI

Open Access Dissertations

2016

\title{
Minimal Cantor Sets: The Combinatorial Construction of Ergodic Families and Semi-Conjugations
}

\author{
Erin Denette \\ University of Rhode Island, edenette@uri.edu
}

Follow this and additional works at: https://digitalcommons.uri.edu/oa_diss

\section{Recommended Citation}

Denette, Erin, "Minimal Cantor Sets: The Combinatorial Construction of Ergodic Families and SemiConjugations" (2016). Open Access Dissertations. Paper 436.

https://digitalcommons.uri.edu/oa_diss/436

This Dissertation is brought to you for free and open access by DigitalCommons@URI. It has been accepted for inclusion in Open Access Dissertations by an authorized administrator of DigitalCommons@URI. For more information, please contact digitalcommons-group@uri.edu. 
MINIMAL CANTOR SETS: THE COMBINATORIAL CONSTRUCTION OF ERGODIC FAMILIES AND SEMI-CONJUGATIONS

BY

ERIN DENETTE

A DISSERTATION SUBMITTED IN PARTIAL FULFILLMENT OF THE REQUIREMENTS FOR THE DEGREE OF DOCTOR OF PHILOSOPHY

IN

MATHEMATICS

UNIVERSITY OF RHODE ISLAND

2016 


\title{
DOCTOR OF PHILOSOPHY DISSERTATION \\ $\mathrm{OF}$ \\ ERIN DENETTE
}

\section{APPROVED:}

Dissertation Committee:

\author{
Major Professor Araceli Bonifant \\ Orlando Merino \\ Mercedes Rivero-Hudec \\ Nasser H. Zawia \\ DEAN OF THE GRADUATE SCHOOL
}

\section{UNIVERSITY OF RHODE ISLAND}




\begin{abstract}
Combinatorially obtained minimal Cantor sets are acquired as the inverse limit of certain directed topological graphs where specific nonnegative integer matrices, called winding matrices, are used to describe the projection between each graph.

Examples of non-uniquely ergodic combinatorially obtained minimal Cantor sets first appeared in the 2006 article Algebraic topology for minimal Cantor sets of Gambaudo and Martens and are constructed using winding matrices whose entries grow "fast enough." In this work, we will introduce families of minimal Cantor sets which may be combinatorially obtained in such a way that the corresponding winding matrices possess unbounded entries given by explicit sequences of nonnegative integers. For each of these families, the growth rate needed to achieve either unique or non-unique ergodicity will be specifically addressed and the result will be applied to the case of minimal Cantor sets corresponding to Lorenz maps.

We will explore the construction of topological semi-conjugations between combinatorially obtained minimal Cantor sets. Theorems guaranteeing the existence of a topological semi-conjugation between specific families of these sets will be proved and utilized to introduce examples possessing additional intriguing properties. We will also show that there exist both finitely and infinitely nonuniquely ergodic minimal Cantor sets semi-conjugated to an adding machine in such a way that the semi-conjugation map is almost-everywhere injective with respect to the unique ergodic invariant probability measure on the adding machine. Furthermore, we will prove that this construction can be realized by the dynamical system $(\omega(c), q)$ where $q$ is a logistic unimodal map with critical point $c$ and omega-limit set $\omega(c)$.
\end{abstract}




\section{ACKNOWLEDGMENTS}

I would like to begin by thanking my major advisor, Dr. Araceli Bonifant, who has gone above and beyond that which is required from her as a teacher and mentor. I am truly grateful for the opportunities she has provided, her continued support, her friendship, and her tireless commitment to my development as a mathematician and to the research contained within this dissertation.

I would also like to thank Dr. Marco Martens of Stonybrook University for generously sharing his research ideas, expertise, and time. Without Dr. Martens' selfless involvement, this dissertation would not be possible.

Dr. Orlando Merino, Dr. Mercedes Rivero-Hudec, Dr. Mustafa Kulenović, and Dr. Liliana Gonzalez graciously served on my committee. I would like to thank them along with Dr. Michael Barrus and Dr. Thomas Sharland for their questions, comments, and suggestions. I deeply appreciate the time they have given to improve this work.

I owe a separate thanks to Dr. Merino, whom I hold in the highest regard as a mathematician, educator, mentor, and person. I appreciate the refreshingly honest, sometimes humorous, and always patient advise, support, and caring he has provided throughout my graduate career.

I would like to thank the University of Rhode Island Mathematics Department for the abundance of opportunities over the past six years, and I would especially like to thank Dr. Kulenović for involving me in his research.

I would not be the mathematician, educator, or person I am today without those peers from my undergraduate and graduate studies who created a community of camaraderie and encouragement. For this and so much more, I thank them.

Thanks is also owed to my family and friends for their unfailing faith and support. I would especially like to acknowledge the advisors of Venturing Crew 1 
Gardner and the faculty of the Fitchburg State University Mathematics Department. Most of all, I would like to thank my husband, Alexander Denette, for being both by my side and on my side since long before this journey began.

My last thanks is reserved for my colleague Addie Armstrong. It is a rare thing to find a friend that is willing to take every step with you on the challenging and often uncertain path that is life. I consider myself among the most fortunate of individuals to have had Addie choose to walk with me. 


\title{
DEDICATION
}

\author{
To my dad \\ Master Chief Daniel Farrell (USCG) \\ February 23, 1959 - July 20, 2013 \\ and to my teacher \\ Professor Mark Snyder (Fitchburg State University) \\ July 31, 1949 - May 29, 2015
}




\title{
TABLE OF CONTENTS
}

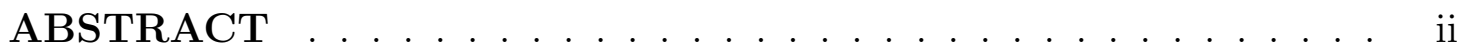

ACKNOWLEDGMENTS ................. . iii

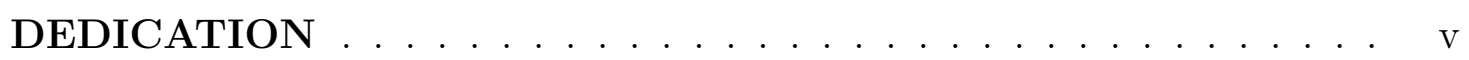

TABLE OF CONTENTS ................... . . vi

LIST OF FIGURES . . . . . . . . . . . . . . . . . . viii CHAPTER
\end{abstract}

1 Introduction and Preliminaries $\ldots \ldots \ldots \ldots \ldots$

1.1 Introduction . . . . . . . . . . . . . . . . . 1

1.2 Combinatorially Obtained Minimal Cantor Sets . . . . . . . 4

1.3 Summary of Results . . . . . . . . . . . . . . . . . . . . . 9

List of References . . . . . . . . . . . . . . . . . . . 13

2 Constructing Families of Ergodic Minimal Cantor Sets . . . . 14

2.1 Introduction . . . . . . . . . . . . . . . . . . 14

2.2 Non-Uniquely Ergodic Minimal Cantor Sets . . . . . . . . 17

2.3 Future Work . . . . . . . . . . . . . . . . . . . . . . . . . . . . . 29

List of References . . . . . . . . . . . . . . . . . 30

3 Almost Everywhere Injective Semi-Conjugations Between Certain Minimal Cantor Sets . . . . . . . . . . . . . 32

3.1 Introduction . . . . . . . . . . . . . . . . 32

3.2 Semi-Conjugations Involving an Adding Machine . . . . . . . 33 


\section{Page}

3.3 Semi-Conjugation Between a Finitely Non-Uniquely Ergodic Minimal Cantor Set and an Adding Machine . . . . . . . . 36

3.4 Semi-Conjugation Between an Infinitely Non-Uniquely Ergodic Minimal Cantor Set and an Adding Machine . . . . . . . . 43

List of References . . . . . . . . . . . . . . . . . . . 4 48

4 Realization Under a Unimodal Map . . . . . . . . . . . . . . 49

4.1 Introduction $\ldots \ldots \ldots \ldots \ldots \ldots \ldots \ldots$

4.2 Kneading Theory and Consequences for Unimodal Maps _. . . 49

4.3 Obtaining the Desired Unimodal Map . . . . . . . . . . . . 54

4.4 Future Work . . . . . . . . . . . . . . . . . . . . . . 64

List of References . . . . . . . . . . . . . . . 65

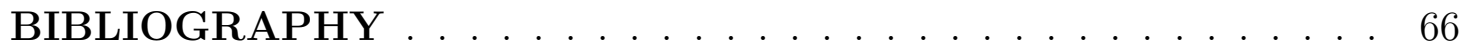




\section{LIST OF FIGURES}

Figure

Page

1 An illustration of the construction of the middle-thirds Cantor set. . 1

2 Plots of $T_{3}$ and $T_{3}^{2}$ on the interval $[0,1]$ for the tent map $T_{3}$ defined in Example 1.2. . . . . . . . . . . . . . . . . . . 3

3 An illustration of a combinatorial cover. Note that $U_{1}, U_{2}$, and $U_{3}$ are the images of the splitting vertex $0_{G}$. The path of $U_{2}, \lambda\left(U_{2}\right)$, is indicated by the dashed edges. . . . . . . . . . . . 7

$4 \quad$ An illustration of Example 1.18. . . . . . . . . . . . . . . . . . 9

$5 \quad$ Plot of a typical Lorenz map on $[0,1] \backslash\{c\} \ldots \ldots \ldots$. . . . . . 19

$6 \quad$ Plots of the tent map $T_{2}$ and a corresponding stunted tent map $\mathcal{S}_{2, p} . \quad 50$

$7 \quad$ Plot of the logistic unimodal map $q_{4}(x)=4 x(1-x) . \quad$. . . . . . 51

8 A possible configuration of the intervals $I_{k}$ and the branches $\mathfrak{o}_{k}: I_{k} \rightarrow[0,1](k=0,1,2)$ of the map $\mathfrak{o}$ as described in Lemma 4.11........................ 56 


\section{CHAPTER 1}

\section{Introduction and Preliminaries}

\subsection{Introduction}

In 1883, Cantor introduced the now well-known middle-thirds Cantor set within a footnote to his paper Über unendliche, lineare Punktmannichfaltigkeiten (see $\left[1\right.$, Part 5]). One method of constructing this set begins by taking $A_{0}=[0,1]$ and then deleting the open middle third of this interval to obtain $A_{1}=\left[0, \frac{1}{3}\right] \cup\left[\frac{2}{3}, 1\right]$. The set $A_{2}$ is formed by deleting the open middle third from each of the disjoint intervals that make up $A_{1}$. In general, the set $A_{n}(n \geq 1)$ is formed by deleting the open middle third from each of the disjoint intervals that make up $A_{n-1}$. The sets $A_{0}, A_{1}, A_{2}$, and $A_{3}$ can been seen in Figure 1. The middle-thirds Cantor set $\mathcal{C}$ is defined as the intersection of the sets $A_{n}$; that is, $\mathcal{C}=\bigcap_{n=0}^{\infty} A_{n}$.

The middle-thirds Cantor set possesses a number of interesting properties. It is clearly nonempty (for instance, it contains 0). It is bounded and, as the intersection of closed sets, it is closed (hence compact). It also contains no intervals since the complement $[0,1] \backslash \mathcal{C}$ has Lebesgue measure one. Furthermore, it is a perfect set since it is a closed set with no isolated points. This can be verified by observing that any $x \in \mathcal{C}$ lies in exactly one of the maximal disjoint intervals of length $\left(\frac{1}{3}\right)^{n}$

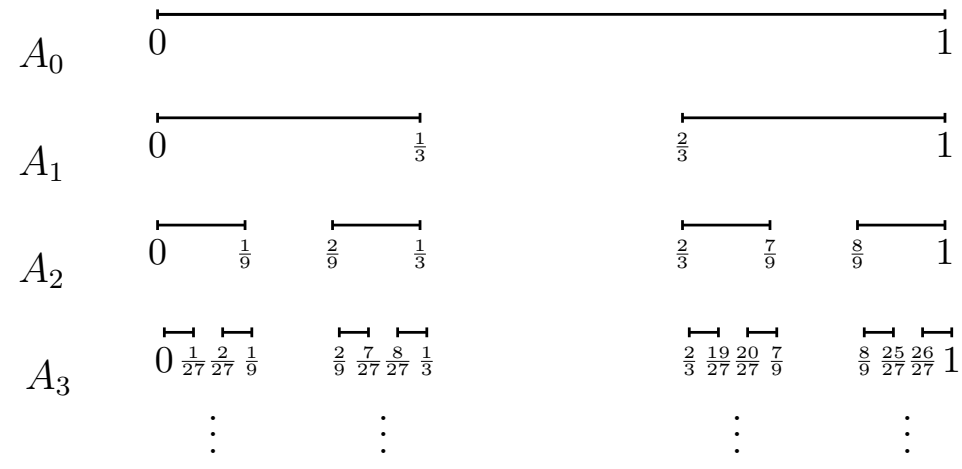

Figure 1. An illustration of the construction of the middle-thirds Cantor set. 
that make up $A_{n}$, say $A_{n}^{x}$. If $x$ is not an endpoint of the interval $A_{n}^{x}$, take $x_{n}$ to be the lefthand endpoint of $A_{n}^{x}$. Otherwise, take $x_{n}$ to be the endpoint of $A_{n}^{x}$ that is not $x$. Since endpoints are never deleted, $x_{n} \in \mathcal{C}$ for each $n$. Moreover, $x_{n} \neq x$ and $\left|x-x_{n}\right| \leq\left(\frac{1}{3}\right)^{n}$ for each $n$. It follows that $x$ is not isolated.

A generalization of the properties of the middle-thirds Cantor set allows for the definition of a Cantor set.

Definition 1.1. A Cantor set is a compact metric space that is totally disconnected and perfect.

Cantor sets arise frequently in the study of dynamical systems, where we are interested in determining the behavior of each point $x \in X$ under iteration of a given function $f: X \rightarrow X$. For $n \in \mathbb{N}$, the notation $f^{n}=f^{\circ n}=\underbrace{f \circ \ldots \circ f}_{n \text { copies of } f}$ is used, so for each $x \in X$, the sequence of interest can be written as

$$
x, f(x), f^{2}(x), f^{3}(x), \ldots
$$

The set of points $\left\{x, f(x), f^{2}(x), f^{3}(x), \ldots\right\}$ is called the orbit of $x$.

Example 1.2. The set of maps $T_{a}: \mathbb{R} \rightarrow \mathbb{R}$ defined by

$$
T_{a}(x)= \begin{cases}a x, & x<\frac{1}{2} \\ a(1-x), & x \geq \frac{1}{2}\end{cases}
$$

for $a>0$ are called tent maps. Consider the tent map $T_{3}$ pictured in Figure 2. All points $x \in(-\infty, 0) \cup(1, \infty)$ tend toward $-\infty$ under iteration of $T_{3}$; such points are said to escape to $-\infty$.

Because of this, the set consisting of those points that do not escape to $-\infty$ under iteration of $T_{3}$ must be a subset of $[0,1]$. It will be shown that this set is precisely the middle-thirds Cantor set.

As can be seen in Figure 2, the set of points $\left\{x: T_{3}(x) \in[0,1]\right\}$ is given by $\left[0, \frac{1}{3}\right] \cup\left[\frac{2}{3}, 1\right]$ and the set of points $\left\{x: T_{3}(x), T_{3}^{2}(x) \in[0,1]\right\}$ is given by 

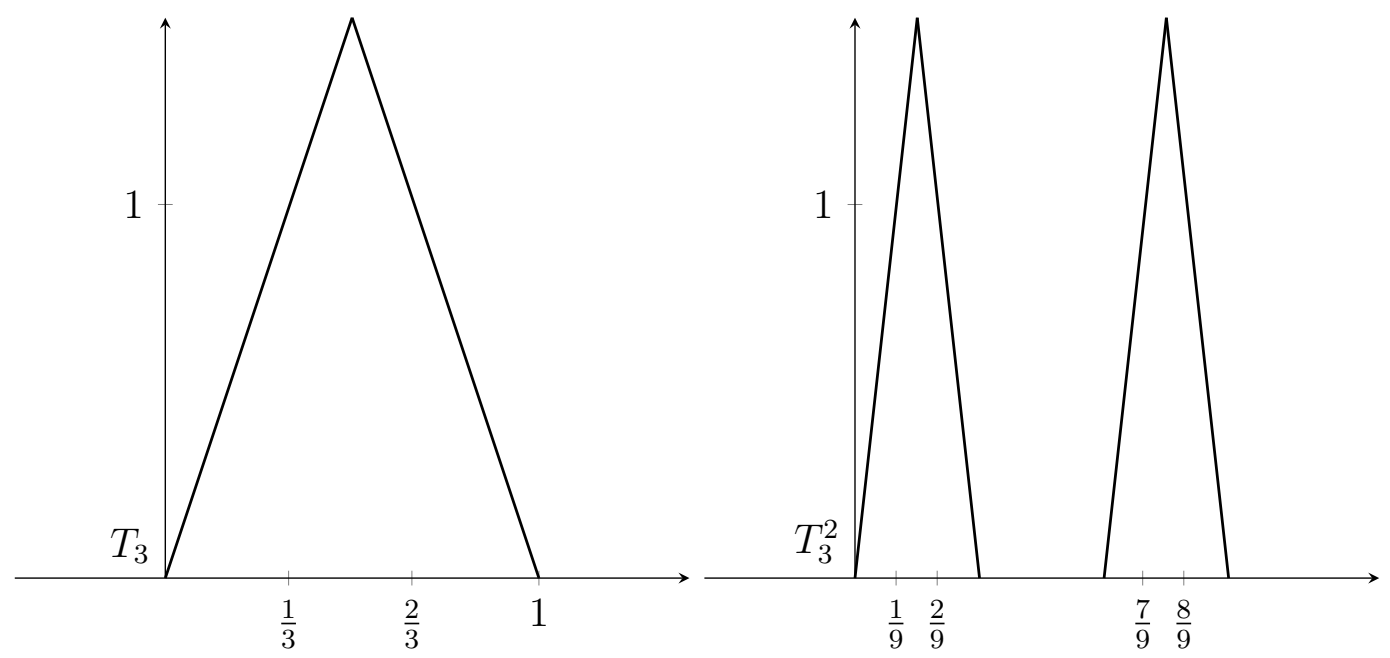

Figure 2. Plots of $T_{3}$ and $T_{3}^{2}$ on the interval $[0,1]$ for the tent map $T_{3}$ defined in Example 1.2.

$\left[0, \frac{1}{9}\right] \cup\left[\frac{2}{9}, \frac{1}{3}\right] \cup\left[\frac{2}{3}, \frac{7}{9}\right] \cup\left[\frac{8}{9}, 1\right]$. Continuing in this fashion, we obtain that the set of points that do not leave the interval $[0,1]$ under any iterate of $T_{3}$ (and hence do not escape to $-\infty$ ) is exactly the middle-thirds Cantor set.

Definition 1.3. A dynamical system $(X, f)$ where $X$ is a compact topological space and $f: X \rightarrow X$ is a continuous map is called minimal if every orbit is dense in $X$. If $X$ is a Cantor set, the system $(X, f)$ is called a minimal Cantor set.

Such minimal systems were originally defined and explored by Birkhoff in 1912 (see [2]), and have garnered a great deal of attention in the literature ever since. Minimal Cantor sets, in particular, often appear in the study of dynamical systems associated with unimodal maps.

Definition 1.4. A unimodal map is a map $u:[0,1] \rightarrow[0,1]$ satisfying $u(0)=u(1)=0$ and for which there exists a point $c \in(0,1)$ (called the critical point) such that $u$ is increasing to the left of $c$ and decreasing to the right of $c$. 
Definition 1.5. For a fixed unimodal map $u$ with critical point $c$, the omega-limit set $\omega(x)$ of a point $x \in[0,1]$ is defined by

$$
\omega(x):=\left\{y \in[0,1]: u^{n_{\ell}}(x) \rightarrow y \text { for some } n_{1}<n_{2}<\ldots\right\}
$$

For a given unimodal map $u:[0,1] \rightarrow[0,1]$ with critical point $c$, the omega limit set $\omega(c)$ is compact and satisfies that $u(\omega(c))=\omega(c)$. The set $\omega(c)$ may be a cycle of intervals, a Cantor set, a finite set, a countable set, or the union of a Cantor set and a countable set. Unimodal maps under which the omega-limit set of the critical point is a minimal Cantor set are known to exist and have been studied widely (see, for instance, $[3,4,5,6,7]$ ). The most well-known examples include the Feigenbaum map (see [8]) and the Fibonacci map (see [5]), which will be described in more detail in Chapter 4.

\subsection{Combinatorially Obtained Minimal Cantor Sets}

In 2006, Gambaudo and Martens gave conditions under which a minimal Cantor set can be obtained via the inverse limit of particular directed topological graphs (see [4]). In their article, the authors introduced the concept of using specific nonnegative integer matrices, called winding matrices, to describe the projection between each of these graphs. This section provides a summary of these and related results.

Definition 1.6. Let $Y_{1}, Y_{2}, \ldots$ be a countable collection of topological spaces and suppose that for each $n \in \mathbb{N}$ there exists a continuous mapping $g_{n}: Y_{n+1} \rightarrow Y_{n}$. The sequence of spaces and mappings $\left\{Y_{n}, g_{n}\right\}_{n=1}^{\infty}$ is called an inverse limit sequence and may be represented by the diagram

$$
Y_{1} \stackrel{g_{1}}{\longleftarrow} Y_{2} \stackrel{g_{2}}{\longleftarrow} \ldots \stackrel{g_{n-1}}{\longleftarrow} Y_{n} \stackrel{g_{n}}{\longleftarrow} Y_{n+1} \stackrel{g_{n+1}}{\longleftarrow} \ldots
$$


For $n<m$, the continuous mapping $g_{m n}: Y_{m} \rightarrow Y_{n}$ is given by

$$
g_{m n}=g_{n} \circ g_{n+1} \circ \ldots \circ g_{m-2} \circ g_{m-1} .
$$

Definition 1.7. The inverse limit of an inverse limit sequence $\left\{Y_{n}, g_{n}\right\}_{n=1}^{\infty}$ is given by

$$
\lim _{g_{n}} Y_{n}=\left\{\left(y_{1}, \ldots, y_{n} \ldots\right) \in \prod_{n=1}^{\infty} Y_{n}: g_{m n}\left(y_{m}\right)=y_{n} \text { for all } n \leq m\right\}
$$

Note that as a subset of the product space $\prod_{n=1}^{\infty} Y_{n}$ carrying the product topology, the inverse limit $Y=\underset{g_{n}}{\lim } Y_{n}$ may be equipped with the corresponding subspace topology.

Definition 1.8. A graph is an ordered pair $G=(\mathcal{V}, \mathcal{E})$ comprised of a set $\mathcal{V}$ of vertices together with a set $\mathcal{E}$ of edges which are unordered, 2-element subsets of $\mathcal{V}$.

We will consider only those graphs for which each edge $\{U, V\}$ satisfies that $U \neq V$

Definition 1.9. A directed graph will consist of a vertex set $\mathcal{V}$, an edge set $\mathcal{E}$, and a function assigning to each edge $e=\{U, V\}=\{V, U\}$ an ordered pair of its associated vertices. If this ordered pair is $(U, V)$ the direction of $e$ is considered to be from $U$ to $V$, while if it is $(V, U)$ the direction of $e$ is considered to be from $V$ to $U$. A directed path in a directed graph is a sequence of edges

$$
e_{1}=\left\{V_{1}, V_{2}\right\}, e_{2}=\left\{V_{2}, V_{3}\right\}, \ldots, e_{n}=\left\{V_{n-1}, V_{n}\right\} \quad(\text { for } n \geq 2)
$$

where all edges are oriented in the same direction.

Gambaudo and Martens introduced an inverse limit sequence in which each topological space consists of a specific type of directed topological graph. 
Definition 1.10. A directed topological graph $G$ is called a combinatorial cover if the following three properties are satisfied:

1. The graph $G$ is finite and the set of vertices carries the discrete topology.

2. The graph $G$ is irreducible (any pair of distinct vertices is connected by a directed path).

3. Except for one vertex $0_{G} \in G$, each vertex of $G$ has exactly one outgoing edge. The vertex $0_{G}$ is called the splitting vertex.

Definition 1.11. In a combinatorial cover $G$, a vertex $U \in G$ is called an image of a vertex $V \in G$ if there is a single directed edge from $V$ to $U$. The shortest directed path from a vertex $V \in G$ to $0_{G}$ is called the path of $V$ and is denoted by $\lambda(V)$. Furthermore, if $U$ is an image of $0_{G}$, then $\lambda(U)$ together with the directed edge from $0_{G}$ to $U$ is called a loop of $G$. When the meaning is clear, the notation $\lambda(U)$ will also be used to denote such a loop.

An illustration of a combinatorial cover and these concepts can be seen in Figure 3.

In order to take the inverse limit involving a sequence of combinatorial covers, it is necessary to define a particular type of mapping between two combinatorial covers.

Definition 1.12. Let $G$ and $H$ be combinatorial covers. A map $\pi: G \rightarrow H$ is called a combinatorial refinement if the following three properties are satisfied:

1. The map $\pi$ preserves the graph structure.

2. The map $\pi$ satisfies that $\pi\left(0_{G}\right)=0_{H}$; that is, the splitting vertex of the graph $G$ is mapped to the splitting vertex of the graph $H$.

3. For each image $1_{H} \in H$ of the splitting vertex $0_{H} \in H$ there 


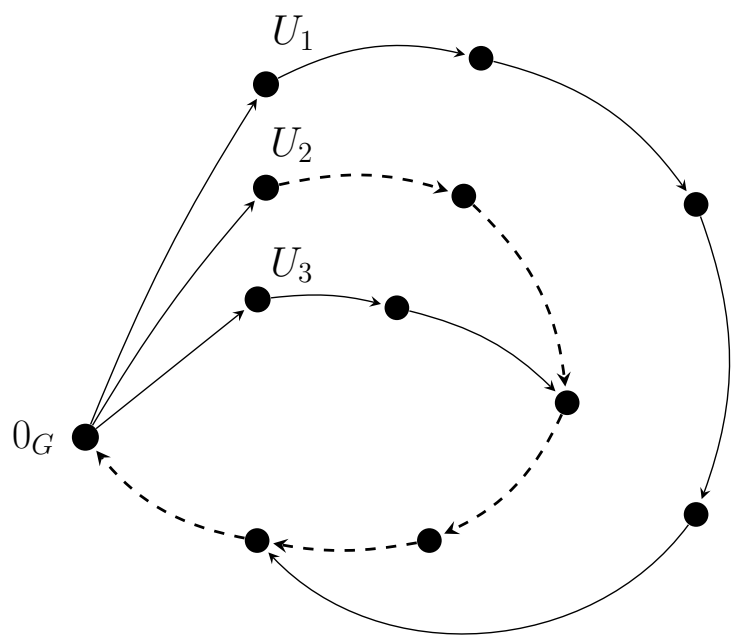

Figure 3. An illustration of a combinatorial cover. Note that $U_{1}, U_{2}$, and $U_{3}$ are the images of the splitting vertex $0_{G}$. The path of $U_{2}, \lambda\left(U_{2}\right)$, is indicated by the dashed edges.

exists an image $V \in G$ of the splitting vertex $0_{G} \in G$ such that

$$
1_{H}=\pi(V)
$$

Lemma 1.13. (Gambaudo \& Martens) Consider an inverse limit sequence consisting of combinatorial covers $\left\{G_{n}\right\}_{n=1}^{\infty}$ and corresponding combinatorial refinements $\left\{\pi_{n}: G_{n+1} \rightarrow G_{n}\right\}_{n=1}^{\infty}$. The corresponding inverse limit is a topological graph

$$
G=\lim _{\pi_{n}} G_{n}
$$

whose edges can be described by a continuous map $f: G \rightarrow G$.

Definition 1.14. Suppose the map $f$ and graph $G$ are as in Lemma 1.13. If the dynamical system $(G, f)$ is a minimal Cantor set it is called a combinatorially obtained minimal Cantor set.

It is only natural to ask what restriction(s), if any, must be placed upon an inverse limit sequence of combinatorial covers and combinatorial refinements in 
order to guarantee that a combinatorially obtained minimal Cantor set will result. The answer to this question requires the definition of a winding matrix.

Definition 1.15. For $G_{n}$ and $G_{n+1}$ combinatorial covers and $\pi_{n}: G_{n+1} \rightarrow G_{n}$ a combinatorial refinement, let $\left\{U_{1}, \ldots, U_{r}\right\}$ be the images of the splitting vertex $0_{G_{n}}$ and $\left\{V_{1}, \ldots, V_{s}\right\}$ be the images of the splitting vertex $0_{G_{n+1}}$. The $r \times s$ matrix $W_{n}$ with entries

$$
w_{i j}=\#\left\{A \in \lambda\left(V_{j}\right): \pi_{n}(A)=U_{i}\right\}
$$

is called the winding matrix corresponding to $\pi_{n}$.

Note that $w_{i j}$ gives the number of distinct vertices in the loop $\lambda\left(V_{j}\right) \in G_{n+1}$ that are projected to the vertex $U_{i} \in G_{n}$ under the combinatorial refinement $\pi_{n}$. Equivalently, $w_{i j}$ gives the number of times the loop $\lambda\left(V_{j}\right) \in G_{n+1}$ is wrapped around the loop $\lambda\left(U_{i}\right) \in G_{n}$ under $\pi_{n}$.

Example 1.16. Suppose that for some $n$ the projection between the combinatorial covers $G_{n+1}$ and $G_{n}$ as pictured in Figure 4 has corresponding winding matrix

$$
W_{n}=\left(\begin{array}{lll}
2 & 1 & 3 \\
1 & 3 & 0
\end{array}\right)
$$

This matrix describes the projection in the following manner: The outermost loop of $G_{n+1}$ is wrapped twice around the outer loop of $G_{n}$ and once around the inner loop of $G_{n}$. The middle loop of $G_{n+1}$ is wrapped once around the outer loop of $G_{n}$ and thrice around the inner loop of $G_{n}$. The innermost loop of $G_{n+1}$ is wrapped thrice around the outer loop of $G_{n}$ and zero times around the inner loop of $G_{n}$.

Theorem 1.17. (Gambaudo \& Martens) Consider an inverse limit sequence consisting of combinatorial covers $\left\{G_{n}\right\}_{n=1}^{\infty}$ and corresponding combinatorial refinements $\left\{\pi_{n}: G_{n+1} \rightarrow G_{n}\right\}_{n=1}^{\infty}$. The dynamical system $(G, f)$ where the map 
$G_{n}$

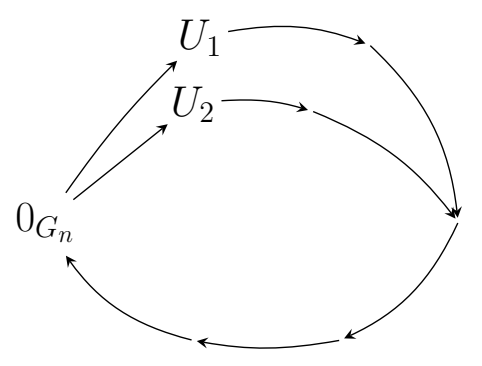

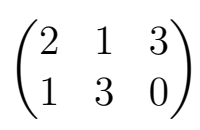

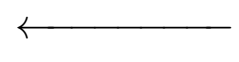

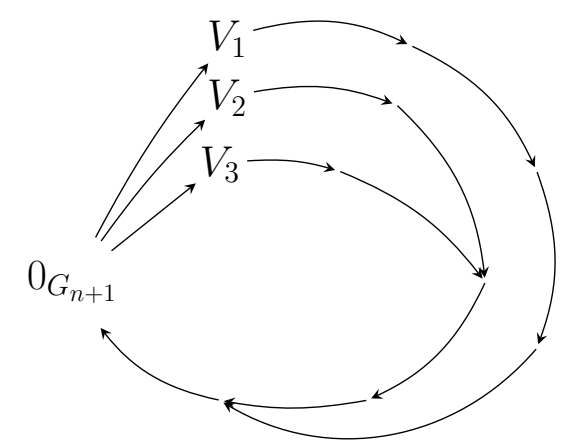

Figure 4. An illustration of Example 1.18.

$f: G \rightarrow G$ is obtained by taking the inverse limit $G=\underset{\pi_{n}}{\lim _{n}} G_{n}$ is a minimal Cantor set if and only if for every $n \in \mathbb{N}$ there exists an $m \in \mathbb{N}$ such that all entries in the first column of the matrix obtained from the product $W_{n} \ldots W_{m}$ are at least 2 .

Example 1.18. Consider a sequence of combinatorial covers $\left\{G_{n}\right\}_{n=1}^{\infty}$ where the combinatorial refinements $\pi_{n}: G_{n+1} \rightarrow G_{n}$ have identical corresponding winding matrices given by $W=\left(\begin{array}{ll}1 & 1 \\ 1 & 0\end{array}\right)$ for each $n$. Since $W^{3}=\left(\begin{array}{ll}3 & 2 \\ 2 & 1\end{array}\right)$, Theorem 1.17 guarantees that the map $f: G \rightarrow G$ obtained by taking the inverse limit $G=\lim _{\pi_{n}} G_{n}$ is a minimal Cantor set.

Now suppose that in the above scenario the winding matrix $W$ is given by $\left(\begin{array}{ll}1 & 1 \\ 0 & 1\end{array}\right)$ instead. It can be shown that for any $n, W^{n}$ is of the form $\left(\begin{array}{ll}* & * \\ 0 & *\end{array}\right)$. Because of the zero in the bottom left corner, Theorem 1.17 guarantees that the map $f: G \rightarrow G$ obtained by taking the inverse $\operatorname{limit} G=\underset{\pi_{n}}{\lim } G_{n}$ is not a minimal Cantor set.

\subsection{Summary of Results}

The study of a given dynamical system $(X, f)$ is usually conducted from a perspective that "makes sense" for the space $X$ and map $f$ under consideration. If 
$X$ is a $C^{r}$-differentiable manifold and $f$ is a $C^{r}$-diffeomorphism, the system can be approached from the point of view of differentiable dynamics. If $X$ is a topological space and $f$ is continuous, topological dynamics may be utilized. If $X$ is a measure space and $f$ measurable, the methods of measurable dynamics may be used.

In the case of a combinatorially obtained minimal Cantor set $(X, f)$, the space $X$ is both a topological space and a measure space, and the map $f$ is both continuous and measurable. The theory of topological dynamics and the theory of measurable dynamics both apply, and for this reason the topological and measuretheoretical properties of such a system are of great interest.

The remaining chapters of this work are concerned with exploring the measuretheoretical property of ergodicity and the construction of topological semi-conjugations in regards to combinatorially obtained minimal Cantor sets.

Definition 1.19. Given a probability space $(X, \Sigma, \mu)$, a map $f: X \rightarrow X$ is called a measure-preserving transformation if $f$ is measurable and $\mu\left(f^{-1}(A)\right)=\mu(A)$ for all $A \in \Sigma$. Furthermore, $f$ is said to be ergodic if for every $A \in \Sigma$ with $f^{-1}(A)=A$ either $\mu(A)=0$ or $\mu(A)=1$. In this case the measure $\mu$ is called an ergodic measure.

Definition 1.20. Let $X$ and $Y$ be topological spaces and let $f: X \rightarrow X$ and $g: Y \rightarrow Y$ be continuous functions. The map $f$ is said to be topologically semiconjugate to the map $g$ if there exists a continuous surjection $p: Y \rightarrow X$ such that $f \circ p=p \circ g$. The map $p$ is called a topological semi-conjugation.

The study of ergodicity is important as ergodic theory allows us to come to a better understanding of how predictable an unpredictable dynamical system can be. Combinatorially obtained minimal Cantor sets are known to carry a set of ergodic invariant probability measures and non-uniquely ergodic minimal Cantor sets are known to exist. Both the paper of Gambaudo and Martens (see [4]) and 
the 2011 doctoral thesis of Winckler (see [9]) contain examples of combinatorially obtained minimal Cantor sets that are not uniquely ergodic. In each of these examples, the authors rely upon winding matrices whose unbounded entries grow "fast enough" but do not address the necessary growth rate. Chapter 2 will provide details concerning these two examples before going on to establish the following main result on the growth rate needed to guarantee either unique or non-unique ergodicity for a specific family of combinatorially obtained minimal Cantor sets.

Theorem 2.7. Let $a, b, m, p \in \mathbb{Z}^{+}$be such that $a>b$ and $m \geq 2$. Then the minimal Cantor set corresponding to the sequences of $m \times m$ winding matrices $\left\{W_{n}\right\}_{n=1}^{\infty}$, with

$$
W_{n}=\left(\begin{array}{cccc}
a n^{p} & b & \cdots & b \\
b & a n^{p} & \ddots & \vdots \\
\vdots & \ddots & \ddots & b \\
b & \ldots & b & a n^{p}
\end{array}\right)
$$

is uniquely ergodic when $p=1$ and is not uniquely ergodic when $p>1$. In the second case, there are in fact $m$ ergodic invariant probability measures.

The remainder of Chapter 2 consists of theorems that establish the ergodicity of families of minimal Cantor sets that are combinatorially obtained from winding matrices with a similar structure to those that appear in Theorem 2.7. These results are then applied in the case of a specific type of map.

Chapters 3 and 4 use the results of Gambaudo and Martens to explore the construction of topological semi-conjugations between minimal Cantor sets, which is a topic of interest since such semi-conjugations are known to preserve a variety of dynamical properties. In Chapter 3, theorems guaranteeing the existence of a topological semi-conjugation between specific families of combinatorially obtained minimal Cantor sets will be established and utilized to prove the following main results. 
Theorem 3.12. The minimal Cantor set that is combinatorially obtained from the sequence of $m \times m(m \geq 3)$ winding matrices $\left\{W_{n}\right\}_{n=1}^{\infty}$, with

$$
W_{n}=\left(\begin{array}{cccccc}
a_{n} & a_{n} & a_{n} & \ldots & & a_{n} \\
n a_{n}+1 & (m-1) n a_{n} & 2 & 2 & \ldots & 2 \\
n a_{n}+1 & 2 & (m-1) n a_{n} & 0 & \ldots & 0 \\
n a_{n} & 0 & 0 & \ddots & \ddots & \vdots \\
\vdots & & & \ddots & & 0 \\
n a_{n} & 0 & \ldots & & 0 & (m-1) n a_{n}
\end{array}\right)
$$

is finitely non-uniquely ergodic for an appropriately chosen sequence of integers $\left\{a_{n}\right\}_{n=1}^{\infty}$. For such a sequence $\left\{a_{n}\right\}_{n=1}^{\infty}$, this minimal Cantor set can be semiconjugated to a specific uniquely ergodic minimal Cantor set in such a way that the semi-conjugation map is almost everywhere injective with respect to the unique ergodic probability measure on the latter minimal Cantor set.

Theorem 3.18. The minimal Cantor set that is combinatorially obtained from the sequence of $m_{n} \times\left(m_{n}+1\right)\left(m_{n} \geq 3\right.$ and $\left.m_{n}=n+2\right)$ winding matrices $\left\{W_{n}\right\}_{n=1}^{\infty}$, with

$$
W_{n}=\left(\begin{array}{ccccccc}
a_{n} & a_{n} & & \ldots & & a_{n} & a_{n} \\
\frac{1}{m_{n}-1} n a_{n}+1 & n a_{n} & 2 & 2 & \ldots & 2 & 2 \\
\frac{1}{m_{n}-1} n a_{n}+1 & 2 & n a_{n} & 0 & \ldots & 0 & 0 \\
\frac{1}{m_{n}-1} n a_{n} & 0 & 0 & \ddots & \ddots & \vdots & \vdots \\
\vdots & \vdots & & \ddots & & 0 & 0 \\
\frac{1}{m_{n}-1} n a_{n} & 0 & \ldots & & 0 & n a_{n} & n a_{n}
\end{array}\right)
$$

is infinitely non-uniquely ergodic for an appropriately chosen sequence of integers $\left\{a_{n}\right\}_{n=1}^{\infty}$. For such a sequence $\left\{a_{n}\right\}_{n=1}^{\infty}$, this minimal Cantor set can be semiconjugated to a specific uniquely ergodic minimal Cantor set in such a way that the semi-conjugation map is almost everywhere injective with respect to the unique ergodic probability measure on the latter minimal Cantor set.

Finally, the main result of Chapter 4 proves that the non-uniquely ergodic minimal Cantor set of Theorem 3.12 can be taken to be $(\omega(c), q)$, where 
$q:[0,1] \rightarrow[0,1]$ is a particular type of quadratic unimodal map with critical point $c$.

Theorem 4.24. Given $m \geq 2$, there exists a quadratic map $q$ with critical point c satisfying that $\omega(c)$ is a non-uniquely ergodic minimal Cantor set possessing $m$ measures. Moreover, this quadratic maps satisfies that $(\omega(c), q)$ can be semiconjugated to a specific uniquely ergodic minimal Cantor set in such a way that the semi-conjugation map is almost everywhere injective with respect to the unique ergodic probability measure on the latter minimal Cantor set.

\section{List of References}

[1] G. Cantor, "Über unendliche, lineare Punktmannichfaltigkeiten," Math. Ann., vol. 21, pp. 545-591, 1883.

[2] G. Birkhoff, "Quelques théorèmes sur le mouvement des systèmes dynamiques," Bull. Soc. Math. France, vol. 40, pp. 305-323, 1912.

[3] M. Martens, "Distortion results and invariant Cantor sets of unimodal maps," Ergodic Theory and Dynamical Systems, vol. 14, pp. 331-349, 1992.

[4] J. Gambaudo and M. Martens, "Algebraic topology for minimal Cantor sets," Annales Henri Poincaré, vol. 7, pp. 423-446, 2006.

[5] J. Milnor and W. Thurston, "On iterated maps of the interval," in Dynamical Systems, ser. Lecture Notes in Mathematics, J. Alexander, Ed. Springer Berlin Heidelberg, 1988, vol. 1342, pp. 465-563.

[6] H. Bruin, "Minimal Cantor systems and unimodal maps," Journal of Difference Equations and Applications, vol. 9(3-4), pp. 305-318, 2003.

[7] L. Alvin, "Uniformly recurrent sequences and minimal Cantor omega-limit sets," Fund. Math., vol. 231, pp. 273-284, 2015.

[8] W. de Melo and S. van Strien, One-Dimensional Dynamics. Springer, 1993.

[9] B. Winckler, "Renormalization of Lorenz maps," Ph.D. dissertation, Royal Institute of Techology, Sweden, 2011. 


\section{CHAPTER 2}

\section{Constructing Families of Ergodic Minimal Cantor Sets}

\section{$2.1 \quad$ Introduction}

The number of ergodic invariant probability measures that a given combinatorially obtained minimal Cantor set carries can be investigated by using the winding matrices associated with it. A brief overview of the background involved in this process is outlined below; all details are provided by Gambaudo and Martens in [1].

Let $(X, f)$ be a combinatorially obtained minimal Cantor set with inverse limit representation $X=\lim _{\pi_{n}} X_{n}$ where each combinatorial cover $X_{n}$ possesses $d_{n}$ loops. Let $\mathcal{M}(X)$ be the space of signed invariant measures on $(X, f)$ where $\Sigma$ is the $\sigma$-algebra on $X$. The space of signed measures on $X_{n}$ has $\sigma$-algebra $\Sigma_{n}$ generated by the elements of $X_{n}$. Let $U_{1}, \ldots, U_{d_{n}}$ be the $d_{n}$ images of the splitting vertex $0_{X_{n}}$ so that the loops of $X_{n}$ are $\lambda\left(U_{1}\right), \ldots, \lambda\left(U_{d_{n}}\right)$. For $j=1, \ldots, d_{n}$, the loop $\lambda\left(U_{j}\right)$ carries an invariant measure $\nu_{j}^{n}: \Sigma_{n} \rightarrow \mathbb{R}$ with $\lambda\left(U_{j}\right)$ as support and $\nu_{j}^{n}(A)=1$ if and only if $A \in \lambda\left(U_{j}\right)$.

Let $H_{1}\left(X_{n}\right)$ be the vector space generated by $\nu_{1}^{n}, \ldots, \nu_{d_{n}}^{n}$. For a given measure $\mu \in \mathcal{M}(X)$, the inclusion $p_{n}: X \rightarrow X_{n}$ induces a map $\left(p_{n}\right)_{*}: \mathcal{M}(X) \rightarrow H_{1}\left(X_{n}\right)$ where $\left(p_{n}\right)_{*}(\mu)$ is the measure obtained when $\Sigma$ is restricted to $\Sigma_{n}$. Similarly, the maps $\pi_{n}: X_{n+1} \rightarrow X_{n}$ induce a linear map $\left(\pi_{n}\right)_{*}: H_{1}\left(X_{n+1}\right) \rightarrow H_{1}\left(X_{n}\right)$. When represented using the bases $\left\{\nu_{1}^{n+1}, \ldots, \nu_{d_{n+1}}^{n+1}\right\}$ and $\left\{\nu_{1}^{n}, \ldots, \nu_{d_{n}}^{n}\right\}$, it can be shown that $\left(p_{n}\right)_{*}=W_{n}$, where $W_{n}$ is the winding matrix corresponding to $\pi_{n}$. This result and the fact that $X=\lim _{\pi_{n}} X_{n}$ leads to the conclusion that $\underset{W_{n}}{\lim } H_{1}\left(X_{n}\right)$ is isomorphic to $\mathcal{M}(X)$.

Let $\mathcal{I}(X) \subset \mathcal{M}(X)$ be the set of invariant measures of $f$ and let $\mathcal{P}(X) \subset \mathcal{I}(X)$ 
consist of the corresponding set of probability measures. Define

$$
H_{1}^{+}\left(X_{n}\right)=\left\{\sum_{j=1}^{d_{n}} \alpha_{j} \nu_{j}^{n}: \alpha_{j} \geq 0\right\} .
$$

Then $I\left(X_{n}\right)=\bigcap_{j=n+1}^{\infty} W_{n} \ldots W_{j}\left(H_{1}^{+}\left(X_{j}\right)\right)$ is the set of invariant measures associated with $X_{n}$ and the corresponding set of probability measures is denoted by $P\left(X_{n}\right)$. Note that each $I\left(X_{n}\right)$ is a cone in $H_{1}\left(X_{n}\right), W_{n}\left(I\left(X_{n+1}\right)\right)=I\left(X_{n}\right)$, and $\lim _{W_{n}} I\left(X_{n}\right)$ is well-defined. Hence $\mathcal{I}(X)$ is isomorphic to $\lim _{\overleftarrow{W_{n}}} I\left(X_{n}\right)$ and $\mathcal{P}(X)$ is isomorphic to $\lim _{\overleftarrow{W_{n}}} P\left(X_{n}\right)$

The proofs of Theorems 2.3 and 2.4 illustrate how this theory can be used to determine the number of ergodic probability measures carried by a given combinatorially obtained minimal Cantor set, and have been included below for this reason.

Definition 2.1. A minimal Cantor set is said to have bounded combinatorics if it can be combinatorially obtained in such a way that the entries of each winding matrix $W_{n}$ are positive and the size and entries of these matrices are uniformly bounded.

Example 2.2. The minimal Cantor set obtained from the sequence of winding matrices $\left\{W_{n}\right\}_{n=1}^{\infty}$ where

$$
W_{n}=\left(\begin{array}{cc}
1 & 1 \\
1 & 2^{n}(\bmod 32)
\end{array}\right)
$$

for each $n$ is of bounded combinatorics since the largest entry that appears in any matrix is 16 and each matrix is $2 \times 2$. However, the minimal Cantor set obtained from the sequence of winding matrices $\left\{W_{n}\right\}_{n=1}^{\infty}$ where

$$
W_{n}=\left(\begin{array}{cc}
1 & 1 \\
1 & 2^{n}
\end{array}\right)
$$

for each $n$ is not of bounded combinatorics since matrix entries are unbounded $\left(2^{n}\right.$ approaches infinity as $n$ approaches infinity). 
Theorem 2.3. (Gambaudo \& Martens) Let $(X, f)$ where $X \underset{\pi_{n}}{\lim } X_{n}$ be a combinatorially obtained minimal Cantor set. If for each $n \in \mathbb{N}$ the number of loops in $X_{n}$ is uniformly bounded by $d$ then $f$ has at most d ergodic invariant probability measures.

Proof. Since the number of loops $d_{n}$ in $X_{n}$ is uniformly bounded by $d$ for each $n$, we may assume without loss of generality that $d_{n}=d$ for each $n$. For $j=1, \ldots, d$, let $t_{j}^{n}$ be the number of vertices in the loop $\lambda\left(U_{j}\right)$. The basis measures $\nu_{1}^{n}, \ldots, \nu_{d}^{n}$ of $H_{1}\left(X_{n}\right)$ may be normalized to probability measures $\mu_{1}^{n}, \ldots, \mu_{d}^{n}$ by setting $\mu_{j}^{n}=\frac{1}{t_{j}^{n}} \nu_{j}^{n}$ for $j=1, \ldots d$.

Let $P_{m}\left(X_{m}\right) \subset H_{1}^{+}\left(X_{m}\right)$ be the set of probability measures and $P_{n}^{m}=W_{n m}\left(P_{m}\right)$. Since $P_{m}$ is the convex hull of the $\left\{\mu_{j}^{n}\right\}, P_{n}^{m}$ is the convex hull of the measures $\mu_{j}^{n m}=W_{n m}\left(\mu_{j}^{m}\right)$. By taking a subsequence it can be assumed that the measures $\mu_{j}^{n m}$ converge to measures $\mu_{j} \in P_{n}$ for $j=1, \ldots d$. But $P\left(X_{n}\right)=\bigcap P_{n}^{m}$, so $P\left(X_{n}\right)$ is the convex hull of the measures $\left\{\mu_{j}: j=1, \ldots, d\right\}$ and therefore it is the convex hull of at most $d$ points (and hence has at most $d$ extreme points and, consequently, at most $d$ ergodic measures).

For the sake of contradiction, suppose $(X, f)$ carries more than $d$ ergodic measures. For $n$ sufficiently large, the projection of these ergodic measures would be distinct extremal points of $P\left(X_{n}\right)$, which has at most $d$ extremal points. This contradiction yields the desired result.

Theorem 2.4. (Gambaudo \& Martens) Let $(X, f)$ be a combinatorially obtained minimal Cantor set. If $(X, f)$ is of bounded combinatorics then $(X, f)$ is uniquely ergodic.

Proof. It suffices to show that $\mathcal{I}(X)$ is one-dimensional. To this end, note that 
the hyperbolic distance between two points $x, y \in H_{1}^{+}\left(X_{n}\right)$ is

$$
\operatorname{hyp}(x, y)=-\ln \frac{(m+\ell)(m+r)}{\ell r} \text {. }
$$

Here, $m$ is the length of the line segment $[x, y]$ and $\ell, r$ are the length of the connected components of $T \backslash[x, y]$ where $T$ is the largest line segment in $H_{1}^{+}\left(X_{n}\right)$ containing $[x, y]$. Positive matrices contract the hyperbolic distance on positive cones. Since $X$ is of bounded combinatorics, the winding matrices $W_{n}$ are uniformly bounded in size and entries. This implies that the contraction is uniform and

$$
I\left(X_{n}\right)=\bigcap_{j=n+1}^{\infty} W_{j n}\left(H_{1}^{+}\left(X_{n}\right)\right)
$$

is one-dimensional for each $n$. Since $\mathcal{I}(X)$ is isomorphic to $\lim _{W_{n}} I\left(X_{n}\right)$, it is also one dimensional. As $\mathcal{P}(X)$ is a subset of $\mathcal{I}(X)$, it is too one-dimensional and hence the map $f$ has only one ergodic invariant probability measure.

\subsection{Non-Uniquely Ergodic Minimal Cantor Sets}

We are interested in constructing combinatorially obtained minimal Cantor sets that are either uniquely ergodic but not of bounded combinatorics or nonuniquely ergodic. In either case, Theorem 2.4 stipulates that this can only be done if the minimal Cantor set does not have bounded combinatorics; that is, the winding matrices $W_{n}$ must be unbounded in size, possess unbounded entries, or both. To simplify computations, we will focus solely on those minimal Cantor sets that can be combinatorially obtained from square winding matrices of a fixed size. The next proposition, which is a consequence of Theorem 2.3, specifies the maximum number of ergodic invariant probability measures that such a combinatorially obtained minimal Cantor set can possess.

Proposition 2.5. Let $m \in \mathbb{N}$ be fixed and let $(X, f)$ be a minimal Cantor set that is combinatorially obtained from a sequence of winding matrices $\left\{W_{n}\right\}_{n=1}^{\infty}$ where each 
matrix is of size $m \times m$. Then $(X, f)$ has at most $m$ ergodic invariant probability measures.

Proof. Consider the corresponding inverse limit $X=\underset{\pi_{n}}{\lim } X_{n}$ of $f$. For each $n$, the winding matrix $W_{n}$ describes the projection from the combinatorial cover $X_{n+1}$ to the combinatorial cover $X_{n}$. That is, for the images $\left\{V_{j}\right\}$ of the splitting vertex $0_{X_{n+1}}$ in $X_{n+1}$ and the images $\left\{U_{i}\right\}$ of the splitting vertex $0_{X_{n}}$ in $X_{n}$, an entry $w_{i j} \in W_{n}$ is the number of times the loop $\lambda\left(V_{j}\right)$ of $X_{n+1}$ passes through the loop $\lambda\left(U_{i}\right)$ of $X_{n}$. Since each $W_{n}$ is an $m \times m$ matrix, each $X_{n}$ must possess $m$ loops. It is now clear that the number of loops in each $X_{n}$ is uniformly bounded by $m$, and the result follows from Theorem 2.3.

Definition 2.6. A Lorenz map on $[0,1] \backslash\{c\}$ is a monotone increasing differentiable map $f$ on $[0, c) \cup(c, 1]$ with $f(0)=0$ and $f(1)=1$ and for which there exists a $c \in(0,1)$ satisfying that $f^{\prime}(x) \rightarrow 0$ as $x \rightarrow c$.

An illustration of a typical Lorenz map can be seen in Figure 5.

In [2], Winckler showed that there exist minimal Cantor sets under Lorenz maps which can obtained from the sequence of winding matrices $\left\{W_{n}\right\}_{n=1}^{\infty}$, where

$$
W_{n}=\left(\begin{array}{cc}
1 & b_{n} \\
a_{n} & 1
\end{array}\right)
$$

and $\left\{a_{n}\right\}_{n=1}^{\infty},\left\{b_{n}\right\}_{n=1}^{\infty}$ are unbounded sequences of integers. Winckler argued that when these unbounded sequences of integers grow quickly enough, it can be guaranteed that the corresponding minimal Cantor set will not be uniquely ergodic and will in fact possesses two ergodic invariant probability measures. When these additional growth conditions are not met, the result is a uniquely ergodic minimal Cantor set that is not of bounded combinatorics.

Gambaudo and Martens stated that there in fact exist minimal Cantor sets possessing $d-1$ ergodic invariant probability measures which can be combinato- 


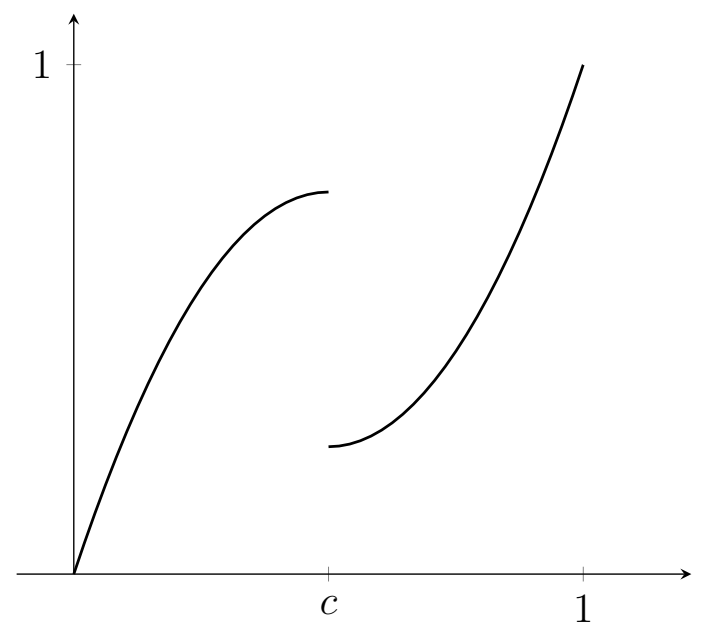

Figure 5. Plot of a typical Lorenz map on $[0,1] \backslash\{c\}$.

rially obtained from combinatorial covers $X_{n}$ which all have $d>1$ loops. Their construction requires the corresponding winding matrices $W_{n}$ to consist of 1's everywhere except for specific entries along the main diagonal, where entries of the second and following columns consist of a large integer $w_{n}$. The result follows from choosing the sequence $\left\{w_{n}\right\}_{n=1}^{\infty}$ to be unbounded and growing sufficiently quickly.

Neither Winckler's Lorenz map example nor the example of Gambaudo and Martens provide insight into exactly how quickly the sequences of unbounded matrix entries must grow in order for non-unique ergodicity to be achieved. The main results in this section will introduce combinatorially obtained minimal Cantor sets whose associated winding matrices possess unbounded entries given by explicit sequences of integers. The specific structure of these winding matrices will allow us to address the precise growth rate needed to guarantee either unique or non-unique ergodicity given a specific additional restriction on the winding matrix entries of Winckler's Lorenz minimal Cantor set example.

Theorem 2.7. Let $a, b, m, p \in \mathbb{Z}^{+}$be such that $a>b$ and $m \geq 2$. Then the minimal Cantor set corresponding to the sequences of $m \times m$ winding matrices 
$\left\{W_{n}\right\}_{n=1}^{\infty}$, with

$$
W_{n}=\left(\begin{array}{cccc}
a n^{p} & b & \cdots & b \\
b & a n^{p} & \ddots & \vdots \\
\vdots & \ddots & \ddots & b \\
b & \ldots & b & a n^{p}
\end{array}\right)
$$

is uniquely ergodic when $p=1$ and is not uniquely ergodic when $p>1$. In the second case, there are in fact $m$ ergodic invariant probability measures.

The proof of this result requires some knowledge regarding the convergence of infinite products.

Lemma 2.8. [3] Let $\left\{a_{n}\right\}_{n=1}^{\infty}$ be a sequence of positive numbers. Then the infinite product $\prod_{n=1}^{\infty}\left(1+a_{n}\right)$ converges if and only if the series $\sum_{n=1}^{\infty} a_{n}$ converges.

Proof of Theorem 2.7. Consider a sequence of winding matrices $\left\{W_{n}\right\}_{n=1}^{\infty}$. As in [2], every invariant measure can be represented by an inverse limit of the sets

$$
\left\{\left(z_{1}, z_{2}, \ldots\right): z_{i}=W_{i} z_{i+1}, z_{i} \in K\right\}
$$

where $K \subset \mathbb{R}^{m}$ is the cone $\left\{\left(x_{1}, \ldots, x_{m}\right): x_{i} \geq 0\right\}$ and hence we should look on the sets

$$
I_{k}=\bigcap_{n>k} W_{k} \ldots W_{n-1} K
$$

We observe that if the limit as $n$ approaches infinity of $W_{k} \ldots W_{n} K$ is of dimension $m$ for each $k$, it will follow that the minimal Cantor set corresponding to $\left\{W_{n}\right\}_{n=1}^{\infty}$ possesses $m$ ergodic invariant probability measures. Similarly, if the limit as $n$ approaches infinity of $W_{k} \cdots W_{n} K$ is a one-dimensional space for each $k$, it will follow that the minimal Cantor set corresponding to $\left\{W_{n}\right\}_{n=1}^{\infty}$ is uniquely ergodic.

Let the main diagonal entries of the matrices $W_{n}$ be given by $a n^{p}$ and all other entries are given by $b$. In seeking a convenient representation of the matrix 
product $W_{k} \ldots W_{n}$ for $n>k$, we observe that each $W_{n}$ is symmetric and exhibits two distinct eigenvalues. The first, $\lambda_{1}^{(n)}=a n^{p}-b$, is of multiplicity $m-1$ and has corresponding eigenvectors $v_{i}$, where $i=1, \ldots, m-1$, consisting of -1 in the first entry, 1 in the $(1+m-i)$-th entry, and zeros elsewhere. The second, $\lambda_{2}^{(n)}=a n^{p}+b(m-1)$, is of multiplicity one and has corresponding eigenvector consisting of all 1's. Hence each $W_{n}$ has spectral decomposition $P D_{n} P^{-1}$, where

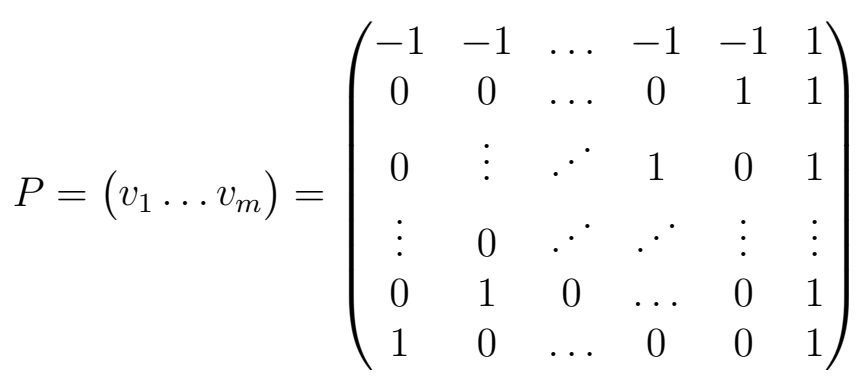

and $D_{n}$ is the diagonal matrix with entries $d_{i i}^{(n)}=\lambda_{1}^{(n)}$ for $i=1, \ldots, m-1$ and $d_{m m}^{(n)}=\lambda_{2}^{(n)}$.

Now for $n>k$,

$$
\begin{aligned}
W_{k n} & =W_{k} \ldots W_{n-1} W_{n} \\
& =\left(P D_{k} P^{-1}\right) \ldots\left(P D_{n-1} P^{-1}\right)\left(P D_{n} P^{-1}\right) \\
& =P D P^{-1}
\end{aligned}
$$

where $D=D_{k} \ldots D_{n}$. The matrix $D$ is diagonal with diagonal entries

$$
d_{i i}=d_{11}^{(k)} \cdot \ldots \cdot d_{11}^{(n)}=\prod_{\ell=k}^{n}\left(a \ell^{p}-b\right)
$$

for $i=1, \ldots, m-1$ and

$$
d_{m m}=d_{m m}^{(k)} \cdot \ldots \cdot d_{m m}^{(n)}=\prod_{\ell=k}^{n}\left(a \ell^{p}+b(m-1)\right) .
$$


It can be shown that

$$
\begin{aligned}
W_{k n} & =P D P^{-1} \\
& =\frac{d_{11}}{m}\left(\begin{array}{cccc}
(m-1)+\frac{d_{m m}}{d_{11}} & \frac{d_{m m}}{d_{11}}-1 & \ldots & \frac{d_{m m}}{d_{11}}-1 \\
\frac{d_{m m}}{d_{11}}-1 & (m-1)+\frac{d_{m m}}{d_{11}} & & \frac{d_{m m}}{d_{11}}-1 \\
\vdots & \ddots & \vdots \\
\frac{d_{m m}}{d_{11}}-1 & \ldots & \frac{d_{m m}}{d_{11}}-1 & (m-1)+\frac{d_{m m}}{d_{11}}-1 \\
\frac{d_{m m}}{d_{11}}-1 & \ldots & (m-1)
\end{array}\right.
\end{aligned}
$$

Let $e_{i}$ be the vector consisting of all zeros except for a single 1 in the $i$-th position. The angle $\theta_{n}$ between $W_{k n} e_{i}$ and $W_{k n} e_{j}$ satisfies that

$$
\cos \theta_{n}=\frac{W_{k n} e_{i} \cdot W_{k n} e_{j}}{\left\|W _ { k n } e _ { i } \left|\left\|\mid W_{k n} e_{j}\right\|\right.\right.}
$$

and

$$
\theta_{n}=\arccos \left(\frac{W_{k n} e_{i} \cdot W_{k n} e_{j}}{\left\|W_{k n} e_{i}\right\|\left\|W_{k n} e_{j}\right\|}\right)
$$

Thus the minimal Cantor set corresponding to $\left\{W_{n}\right\}_{n=1}^{\infty}$ is not uniquely ergodic if for each $k$ there exist some $e_{i}, e_{j}$ with $i \neq j$ where $\left\{\theta_{n}\right\}_{n=1}^{\infty}$ does not converge to zero as $n$ approaches infinity. By equation (6), this is the same as showing that the righthand side of equation (5) does not converge to 1 as $n$ approaches infinity. If no such $e_{i}, e_{j}$ exist for each $k$, then the minimal Cantor set corresponding to $\left\{W_{n}\right\}_{n=1}^{\infty}$ is not uniquely ergodic.

We observe that for any $i \neq j$, equation (5) becomes

$$
\cos \theta_{n}=\frac{2\left((m-1)+\frac{d_{m m}}{d_{11}}\right)\left(\frac{d_{m m}}{d_{11}}-1\right)+(m-2)\left(\frac{d_{m m}}{d_{11}}-1\right)^{2}}{\left((m-1)+\frac{d_{m m}}{d_{11}}\right)^{2}+(m-1)\left(\frac{d_{m m}}{d_{11}}-1\right)^{2}}
$$


Since the only term in the righthand side of equation (7) dependent upon $n$ is

$$
\frac{d_{m m}}{d_{11}}=\prod_{\ell=k}^{n} \frac{a \ell^{p}+b(m-1)}{a \ell^{p}-b}=\prod_{\ell=k}^{n}\left(1+\frac{b m}{a \ell^{p}-b}\right),
$$

the behavior of the righthand side of (7) as $n$ approaches infinity depends upon the behavior of the product (8) as $n$ approaches infinity. Note that $\frac{b m}{a \ell^{p}-b}$ is positive for each $\ell$ and hence by Lemma 2.8 the behavior of the product (8) as $n$ approaches infinity can be determined by the behavior of the series

$$
\sum_{\ell=k}^{\infty} \frac{b m}{a \ell^{p}-b}
$$

If $p=1$, then series (9) diverges by comparison with the harmonic series $\sum_{\ell=k}^{\infty} \frac{1}{\ell}$. Hence the product (8) approaches infinity as $n$ approaches infinity and it can be shown that the righthand side of equation (7) approaches 1 as $n$ approaches infinity. Hence $\left\{\theta_{n}\right\}_{n=1}^{\infty}$ converges to 0 as $n$ approaches infinity and it follows that the minimal Cantor set corresponding to the sequence of winding matrices $\left\{W_{n}\right\}_{n=1}^{\infty}$ is uniquely ergodic.

If $p>1$, then series (9) converges by comparison with the $p$-series $\sum_{\ell=k}^{\infty} \frac{1}{\ell^{p}}$. Hence the product (8) converges to a nonnegative number, say $T$, as $n$ approaches infinity and it can be shown that the righthand side of equation (7) converges to $\frac{T^{2}-1}{T^{2}-1+m}$ as $n$ approaches infinity. This ratio is clearly never equal to 1 for any value of $T$, and so the righthand side of equation (7) does not converge to 1 as $n$ approaches infinity. Hence $\left\{\theta_{n}\right\}_{n=1}^{\infty}$ does not converge to zero as $n$ approaches infinity and it follows that the minimal Cantor set corresponding to the sequence of winding matrices $\left\{W_{n}\right\}_{n=1}^{\infty}$ is not uniquely ergodic. Moreover, since this result holds for any $e_{i}, e_{j}$ where $1 \leq i, j \leq m$ and $i \neq j$, the corresponding minimal Cantor set in fact possesses $m$ ergodic invariant probability measures.

The ergodicity of the above family of minimal Cantor sets was ultimately determined by a comparison test with a $p$-series. This result suggests that for 
$\left\{w_{n}\right\}_{n=1}^{\infty}$ a sequence of integers that eventually grows faster than the sequence $\{n\}_{n=1}^{\infty}$, the minimal Cantor set that is combinatorially obtained from $m \times m$ $(m \geq 2)$ winding matrices $\left\{W_{n}\right\}_{n=1}^{\infty}$ of the form

$$
W_{n}=\left(\begin{array}{cccc}
a w_{n} & b & \cdots & b \\
b & a w_{n} & \ddots & \vdots \\
\vdots & \ddots & \ddots & b \\
b & \cdots & b & a w_{n}
\end{array}\right) ; \text { where } a, b \in \mathbb{Z}^{+} \text {with } a>b
$$

will not be uniquely ergodic. We will further explore this idea by introducing two additional families of non-uniquely ergodic minimal Cantor sets. As will be demonstrated, the ergodicity of the first is ultimately determined by a comparison test involving a geometric series.

Theorem 2.9. Let $a, b, m \in \mathbb{Z}^{+}$be such that $a>b$ and $m \geq 2$. Then the minimal Cantor set corresponding to the sequences of $m \times m$ winding matrices $\left\{W_{n}\right\}_{n=1}^{\infty}$, with

$$
W_{n}=\left(\begin{array}{cccc}
a^{n} & b & \cdots & b \\
b & a^{n} & \ddots & \vdots \\
\vdots & \ddots & \ddots & b \\
b & \cdots & b & a^{n}
\end{array}\right)
$$

is not uniquely ergodic. In fact, there are $m$ ergodic invariant probability measures.

Note that the requirement that $a, b \in \mathbb{Z}^{+}$with $a>b$ ensures that $a \neq 1$. If $a=1$, then each $W_{n}$ would be identical and the corresponding minimal Cantor set would be of bounded combinatorics, and hence uniquely ergodic by Theorem 2.4.

Proof of Theorem 2.9. We will utilize the method that was employed in the proof of Theorem 2.7 .

Let the main diagonal entries of the matrices $W_{n}$ be given by $a^{n}$ and all other entries are given by $b$. Each $W_{n}$ is symmetric and exhibits two distinct eigenvalues. The first, $\lambda_{1}^{(n)}=a^{n}-b$, is of multiplicity $m-1$ and has corresponding eigenvectors 
$v_{i}$, where $i=1, \ldots, m-1$ consisting of -1 in the first entry, 1 in the $(1+m-i)$-th entry, and zeros elsewhere. The second, $\lambda_{2}^{(n)}=a^{n}+b(m-1)$, is of multiplicity one and has corresponding eigenvector consisting of all 1's. Hence each $W_{n}$ has spectral decomposition $P D_{n} P^{-1}$, where $P$ is the matrix given in (3) and $D_{n}$ is the diagonal matrix with entries $d_{i i}^{(n)}=\lambda_{1}^{(n)}$ for $i=1, \ldots, m-1$ and $d_{m m}^{(n)}=\lambda_{2}^{(n)}$. Now for $k<n, W_{k n}=P D P^{-1}$ where $D=D_{k} \cdot \ldots \cdot D_{n}$ is the diagonal matrix with diagonal entries

$$
d_{i i}=d_{11}^{(k)} \cdot \ldots \cdot d_{11}^{(n)}=\prod_{\ell=k}^{n}\left(a^{\ell}-b\right)
$$

for $i=1, \ldots, m-1$ and

$$
d_{m m}=d_{m m}^{(k)} \cdot \ldots \cdot d_{m m}^{(n)}=\prod_{\ell=1}^{n}\left(a^{\ell}+b(m-1)\right) .
$$

The matrix product $W_{k n}$ is given in equation (4).

Let $e_{i}$ be the vector consisting of all zeros except for a single 1 in the $i$-th position. For $i \neq j$, the angle $\theta_{n}$ between $W_{k n} e_{i}$ and $W_{k n} e_{j}$ satisfies equations (5), (6), and (7) above, and it will follow that the minimal Cantor set corresponding to $\left\{W_{n}\right\}_{n=1}^{\infty}$ is not uniquely ergodic if it can be shown that there exist $e_{i}, e_{j}$ with $i \neq j$ such that the sequence of angles $\left\{\theta_{n}\right\}_{n=1}^{\infty}$ does not converge to zero as $n$ approaches infinity. This is equivalent to showing that the righthand side of equation (7) does not converge to 1 as $n$ approaches infinity.

As above, the only term in the righthand side of equation (7) dependent upon $n$ is

$$
\frac{d_{m m}}{d_{11}}=\prod_{\ell=k}^{n} \frac{a^{\ell}+b(m-1)}{a^{\ell}-b}=\prod_{\ell=k}^{n}\left(1+\frac{b m}{a^{\ell}-b}\right) .
$$

and $\frac{b m}{a^{\ell}-b}$ is positive for each $\ell$. Hence by Lemma 2.8, the convergence of the product (10) depends on the convergence of the series $\sum_{\ell=k}^{\infty} \frac{b m}{a^{\ell}-b}$. This series converges by comparison with the geometric series $\sum_{\ell=k}^{\infty} \frac{1}{a^{\ell}}$ (to see this, recall that $a, b \in \mathbb{Z}^{+}$ 
with $a>b$, which implies that $a>2$ ). Hence the righthand side of equation (10) converges to a nonnegative number, say $T$, as $n$ approaches infinity and, as in the proof of Case 1 of Theorem 2.7, it can be shown that the righthand side of equation 7 converges to $\frac{T^{2}-1}{T^{2}-1+m}$ as $n$ approaches infinity. Hence $\left\{\theta_{n}\right\}_{n=1}^{\infty}$ does not converge to zero as $n$ approaches infinity and it follows that the minimal Cantor set corresponding to the sequence of winding matrices $\left\{W_{n}\right\}_{n=1}^{\infty}$ is not uniquely ergodic. Moreover, since this result holds for any $e_{i}, e_{j}$ where $1 \leq i, j \leq m$ and $i \neq j$, the corresponding minimal Cantor set in fact possesses $m$ ergodic invariant probability measures.

Theorem 2.10. Let $a, b, m, p \in \mathbb{Z}^{+}$be such that $a>b$ and $m \geq 2$. Then the minimal Cantor set corresponding to the sequences of $m \times m$ winding matrices $\left\{W_{n}\right\}_{n=1}^{\infty}$, with

$$
W_{n}=\left(\begin{array}{cccc}
a^{n} n^{p} & b & \cdots & b \\
b & a^{n} n^{p} & \ddots & \vdots \\
\vdots & \ddots & \ddots & b \\
b & \ldots & b & a^{n} n^{p}
\end{array}\right)
$$

is not uniquely ergodic. In fact, there are $m$ ergodic invariant probability measures.

Proof. The proof is similar to the proofs of Theorems 2.7 and 2.9.

We wish to apply Theorems 2.7, 2.9, and 2.10 in the case of Lorenz minimal Cantor sets. However, each winding matrix that we have considered has unbounded entries located along the main diagonal while Lorenz minimal Cantor sets have winding matrices of form (2) with unbounded entries located along the minor diagonal. The subsequent theorem will allow us to overcome this obstacle.

Recall that a permutation matrix of size $m \times m$ can be obtained by permuting the rows of the $m \times m$ identity matrix. Left multiplication of a square matrix $A$ 
by a permutation matrix of the same size will permute the rows of $A$ while right multiplication will permute the columns of $A$.

Theorem 2.11. Let $P$ be an $m \times m$ permutation matrix and let the sequence of matrices $\left\{W_{n}\right\}_{n=1}^{\infty}$ be defined as in one of Theorems 2.7, 2.9, or 2.10. Then the ergodicity of the minimal Cantor set corresponding to the sequence of winding matrices $\left\{\widetilde{W}_{n}\right\}_{n=1}^{\infty}$, where

$$
\widetilde{W}_{n}=P W_{n}
$$

for each $n$, is the same as the ergodicity of the minimal Cantor set corresponding to the sequence of winding matrices $\left\{W_{n}\right\}_{n=1}^{\infty}$.

To prove Theorem 2.11, we will make use of the following lemma from the theory of permutation matrices.

Lemma 2.12. [4] An $m \times m$ matrix $A$ commutes with every $m \times m$ permutation matrix if and only if

$$
A=\alpha I+\beta E
$$

where $\alpha, \beta$ are scalars, $I$ is the $m \times m$ identity matrix, and $E$ is the $m \times m$ matrix consisting of all 1's.

Proof of Theorem 2.11. Each matrix in the sequence of winding matrices $\left\{W_{n}\right\}_{n=1}^{\infty}$ defined as in one of Theorem 2.7, Theorem 2.9, or Theorem 2.10 can be written in the form $\alpha I+\beta E$ for some scalars $\alpha$ and $\beta$. Consequently, each $W_{n}$ commutes with the permutation matrix $P$ by Lemma 2.12 .

We now proceed as in the proofs of Theorems 2.7 and 2.9. As before, let $\widetilde{W}_{k n}=\widetilde{W}_{k} \ldots \widetilde{W}_{n}$. Since $\widetilde{W}_{n}=P W_{n}$ for each $n$, we have that

$$
\begin{aligned}
\widetilde{W}_{k n} & =P W_{k} \ldots P W_{n} \\
& =P^{n-k} W_{k} \ldots W_{n}
\end{aligned}
$$


by Lemma 2.12 . The product $W_{k} \ldots W_{n}$ is given in (4) and therefore

$$
\widetilde{W}_{k n}=\frac{d_{11}}{m} P^{n-k} D
$$

where $D$ is the diagonal matrix

$$
\left(\begin{array}{cccc}
(m-1)+\frac{d_{m m}}{d_{11}} & \frac{d_{m m}}{d_{11}}-1 & \ldots & \frac{d_{m m}}{d_{11}}-1 \\
\frac{d_{m m}}{d_{11}}-1 & (m-1)+\frac{d_{m m}}{d_{11}} & & \frac{d_{m m}}{d_{11}}-1 \\
\vdots & \ddots & \vdots \\
\frac{d_{m m}}{d_{11}}-1 & \ldots & \frac{d_{m m}}{d_{11}}-1 & (m-1)+\frac{d_{m m}}{d_{11}}
\end{array}\right)
$$

The $d_{i i}$ depend upon the sequence $\left\{W_{n}\right\}_{n=1}^{\infty}$ in the following manner: if the sequence of winding matrices $\left\{W_{n}\right\}_{n=1}^{\infty}$ is defined as in Theorem 2.7, then the $d_{i i}$ are defined as in Theorem 2.7. A similar result holds if the sequence of winding matrices $\left\{W_{n}\right\}_{n=1}^{\infty}$ is defined as in Theorem 2.9 or Theorem 2.10 instead.

As a power of a permutation matrix, $P^{n-k}$ is itself a permutation matrix. Hence the product $\frac{d_{11}}{m} P^{n-k} D$ will be a scalar multiple of the matrix $D$ with its rows permuted in some manner. Hence for each $n$, the angle $\theta_{n}$ between $\widetilde{W}_{k n} e_{i}$ and $\widetilde{W}_{k n} e_{j}$ for $i \neq j$ will satisfy equations (5), (6), and (7). As a consequence, the ergodicity of the minimal Cantor set corresponding to $\left\{\widetilde{W}_{n}\right\}_{n=1}^{\infty}$ must be the same as the ergodicity of the minimal Cantor set corresponding to the sequence of winding matrices $\left\{W_{n}\right\}_{n=1}^{\infty}$.

Example 2.13. Recall that there exist minimal Cantor sets under Lorenz maps that can be combinatorially obtained via the sequence of winding matrices $\left\{W_{n}\right\}_{n=1}^{\infty}$, where each $W_{n}$ is defined as in equation (2). If we let $a_{n}=b_{n}$, the winding matrices satisfy that

$$
W_{n}=\left(\begin{array}{cc}
1 & a_{n} \\
a_{n} & 1
\end{array}\right)
$$


Let $P$ be the $2 \times 2$ permutation matrix with 1 's along the minor diagonal so that

$$
W_{n}=P\left(\begin{array}{cc}
a_{n} & 1 \\
1 & a_{n}
\end{array}\right)
$$

Now for $a$ and $p$ integers satisfying that $a, p>1$, Theorems 2.7, 2.9, 2.10 and 2.11 guarantee that the Lorenz minimal Cantor set corresponding to the sequence of winding matrices $\left\{W_{n}\right\}_{n=1}^{\infty}$ with

$$
W_{n}=\left(\begin{array}{cc}
1 & a n \\
a n & 1
\end{array}\right)
$$

is uniquely ergodic while the Lorenz minimal Cantor sets corresponding to the sequence of winding matrices $\left\{W_{n}\right\}_{n=1}^{\infty}$ with

$$
W_{n}=\left(\begin{array}{cc}
1 & a n^{p} \\
a n^{p} & 1
\end{array}\right), W_{n}=\left(\begin{array}{cc}
1 & a^{n} \\
a^{n} & 1
\end{array}\right), \text { or } \quad W_{n}=\left(\begin{array}{cc}
1 & a^{n} n^{p} \\
a^{n} n^{p} & 1
\end{array}\right)
$$

are not uniquely ergodic.

\subsection{Future Work}

The structure of each of the winding matrices considered in this chapter allowed for a convenient diagonal representation of the matrix product $W_{k n}$. This is not the case in general, as can be seen by considering the winding matrices introduced in the two conjectures below. These conjectures generalize the example of Gambaudo and Martens described below Proposition 2.5 and are supported by preliminary numerical trials. In each case, however, the eigenvectors corresponding to the winding matrix $W_{n}$ depend upon $n$. This makes obtaining a closed form formula for the matrix product $W_{k n}$ challenging, and it is likely that a different technique will need to be employed to establish the ergodicity of these and other

minimal Cantor sets that are combinatorially obtained from winding matrices that exhibit less symmetry than those that were considered in Theorems 2.7, 2.9, 2.10, and 2.11. 
Conjecture 2.14. Let $a, b, m, p \in \mathbb{Z}^{+}$be such that $a>b$ and $m \geq 2$. Then the minimal Cantor set corresponding to the sequence of $m \times m$ winding matrices $\left\{W_{n}\right\}_{n=1}^{\infty}$, with

$$
W_{n}=\left(\begin{array}{cccccc}
b & b & & \ldots & & b \\
b & \ddots & & & & \\
& & b & \ddots & & \vdots \\
\vdots & & \ddots & a n^{p} & & \\
& & & & \ddots & b \\
b & & \ldots & & b & a n^{p}
\end{array}\right) \text { or } W_{n}=\left(\begin{array}{cccccc}
b & & \ldots & & b & b \\
b & & & & . & b \\
& & . \cdot & b & & \vdots \\
\vdots & & a n^{p} & . & \\
b & . \cdot & & & \\
a n^{p} & b & & \ldots & & b
\end{array}\right),
$$

where are $k \geq 2$ copies of $a n^{p}$ and $m-k$ copies of $b$ along the main or minor diagonal, respectively, is uniquely ergodic when $p=1$ and is not uniquely ergodic when $p>1$. In the second case, there are in fact $k$ ergodic invariant probability measures.

Conjecture 2.15. Let $a, b, m \in \mathbb{Z}^{+}$be such that $a>b$ and $m \geq 2$. Then the minimal Cantor set corresponding to the sequence of $m \times m$ winding matrices $\left\{W_{n}\right\}_{n=1}^{\infty}$, with

$$
W_{n}=\left(\begin{array}{cccccc}
b & b & & \ldots & & b \\
b & \ddots & & & & \\
& & b & \ddots & & \vdots \\
\vdots & & \ddots & a^{n} & & \\
& & & & \ddots & b \\
b & & \ldots & & b & a^{n}
\end{array}\right) \text { or } W_{n}=\left(\begin{array}{cccccc}
b & & \ldots & & b & b \\
b & & & & \cdot & b \\
& & . & b & & \vdots \\
\vdots & & a^{n} & . & \\
b & . & & & \\
a^{n} & b & & \ldots & & b
\end{array}\right) \text {, }
$$

where there are $k \geq 2$ copies of $a^{n}$ and $m-k$ copies of $b$ along the main or minor diagonal, respectively, is not uniquely ergodic and there are in fact $k$ ergodic invariant probability measures.

\section{List of References}

[1] J. Gambaudo and M. Martens, "Algebraic topology for minimal Cantor sets," Annales Henri Poincaré, vol. 7, pp. 423-446, 2006. 
[2] B. Winckler, "Renormalization of Lorenz maps," Ph.D. dissertation, Royal Institute of Techology, Sweden, 2011.

[3] K. Knopp, Theory and Application of Infinite Series. New York: Hafner Publishing Company, 1947.

[4] F. Hohn, Elementary Matrix Algebra (3rd edition). MacMillan, 1973. 


\section{CHAPTER 3}

\section{Almost Everywhere Injective Semi-Conjugations Between Certain Minimal Cantor Sets}

\subsection{Introduction}

For $f: X \rightarrow X$ and $g: Y \rightarrow Y$ continuous functions, $f$ is said to be topologically conjugate to $g$ if there exists a homeomorphism $h: X \rightarrow Y$ such that $h \circ f=g \circ h$; that is, the following diagram commutes.

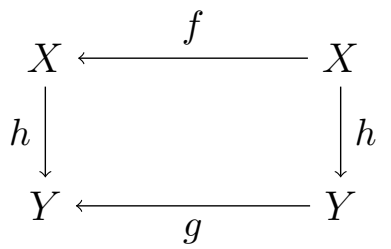

Topological conjugacy plays an important role in studying the dynamics of continuous maps since two topologically conjugate maps are known to have the same dynamical properties.

Recall that for $f$ and $g$ defined as above, $f$ is said to be topologically semiconjugate to $g$ if the function $h$ above is a continuous, surjective function rather than a homeomorphism and the diagram is still commutative. In this case, the map $h$ is called a topological semi-conjugation. Such semi-conjugations also preserve a number of dynamical properties, and if $f$ is semi-conjugate to $g$, it can be said that the dynamics of $(X, f)$ are at least as complicated as the dynamics of $(Y, g)$.

It is well-known that topological semi-conjugations do not preserve measuretheoretical properties. For this reason, it is not necessarily a surprising result that a non-uniquely ergodic minimal Cantor set may be semi-conjugated to a uniquely ergodic minimal Cantor set. In this chapter, it will be shown that for specific combinatorially obtained minimal Cantor sets, the above statement is true even 
when the semi-conjugation map is almost everywhere injective with respect to the ergodic measure associated with the uniquely ergodic minimal Cantor set.

\subsection{Semi-Conjugations Involving an Adding Machine}

We will take as $(Y, g)$ in the definition of a topological semi-conjugation a dynamical system chosen from a particular family of combinatorially obtained minimal Cantor sets called adding machines.

Definition 3.1. Let $\left\{w_{n}\right\}_{n=1}^{\infty}$ be a sequence of positive integers with $w_{n} \geq 2$ for each $n$. An adding machine is a minimal Cantor set that can be combinatorially obtained from a sequence of $1 \times 1$ winding matrices $\left\{W_{n}\right\}_{n=1}^{\infty}$ where $W_{n}=\left(w_{n}\right)$ for each $n$.

Note that adding machines are also frequently referred to as solenoids or odometers in the literature, where they have been studied by many authors, including Collas and Klein, Gambuado and Martens, and Oversteegen (see [1, 2, 3]).

Proposition 3.2. An adding machine is uniquely ergodic.

Proof. Since the dimension of each winding matrix is 1, the adding machine possesses at most one and hence exactly one ergodic invariant probability measure by Proposition 2.5 .

In sections 3.3 and 3.4 , it will be shown that there exist topological semiconjugations between non-uniquely ergodic combinatorially obtained minimal Cantor sets and adding machines that are almost everywhere injective with respect to the unique ergodic measure on the given adding machine. These results require the establishment of a property that will guarantee that certain combinatorially obtained minimal Cantor sets are topologically semi-conjugate to a given adding machine. 
Proposition 3.3. Let $(X, f)$ with $X=\lim _{\pi_{n}} X_{n}$ be a combinatorially obtained minimal Cantor set and let $(Y, g)$ with $Y=\lim _{\psi_{n}} Y_{n}$ be an adding machine. If each of the $d_{n}$ loops of $X_{n}$ possess the same number of vertices as the single loop of $Y_{n}$ then for each $n \in \mathbb{Z}^{+}$there exist continuous, surjective maps $p_{n}: X_{n} \rightarrow Y_{n}$ and $p_{n+1}: X_{n+1} \rightarrow Y_{n+1}$ such that $p_{n} \circ \pi_{n}=\psi_{n} \circ p_{n+1}$; that is, the following diagram commutes.

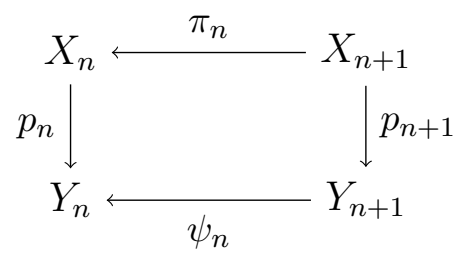

The proof of this proposition requires assigning "labels" to certain vertices in a given combinatorial cover.

Definition 3.4. Let $(X, f)$ with $X=\lim _{\pi_{n}} X_{n}$ be a combinatorially obtained minimal Cantor set. For a vertex $x$ in a given loop of $X_{n}$, the position of $x$ in $X_{n}$, denoted by $\ell_{n}(x)$, is the number of edges in the shortest directed path from the splitting vertex $0_{X_{n}}$ to $x$. The position of the splitting vertex $0_{X_{n}}$ in $X_{n}$ is taken to be 0 .

Note that if the combinatorially cover $X_{n}$ is made up of multiple loops, there may be multiple vertices in a given position. To illustrate this, let $U_{1}, \ldots, U_{d_{n}}$ be the $d_{n}$ distinct images of the splitting vertex $0_{X_{n}}$. The shortest directed path from the splitting vertex to each of these vertices consists of a single directed edge, so each of $U_{1}, \ldots, U_{d_{n}}$ is said to be in position 1 in $X_{n}$.

Proof of Proposition 3.3. For each $n \in \mathbb{N}$, let $t_{n}$ represent the number of vertices in the single loop of $Y_{n}$ and let $s_{n}$ represent in the number of vertices in each of the $d_{n}$ loops of $X_{n}$. By hypothesis, $t_{n}=s_{n}$ for each $n \in \mathbb{N}$. Hence for each 
$n \in \mathbb{N}$, there exists a continuous, surjective map $p_{n}: X_{n} \rightarrow Y_{n}$ that projects each loop of $X_{n}$ once around the single loop of $Y_{n}$ by mapping $x \in X_{n}$ to the unique vertex $y \in Y_{n}$ located in position $\ell_{n}(x)$ in $Y_{n}$. Specifically, this guarantees the existence of continuous, surjective maps $p_{n}: X_{n} \rightarrow Y_{n}$ and $p_{n+1}: X_{n+1} \rightarrow Y_{n+1}$ for each $n \in \mathbb{N}$.

It remains to be shown that for any $x \in X_{n+1}$, the equation

$$
\left(p_{n} \circ \pi_{n}\right)(x)=\left(\psi_{n} \circ p_{n+1}\right)(x)
$$

holds. To prove this, fix $x \in X_{n+1}$. The position of $x$ in $X_{n+1}$ is $\ell_{n+1}(x)$. Now $\pi_{n}(x)$ is a vertex $x^{\prime} \in X_{n}$ in position $\ell_{n+1}(x) \bmod s_{n}$. By definition, $p_{n}\left(x^{\prime}\right)$ is the unique vertex in $Y_{n}$ that is in position $\ell_{n+1}(x) \bmod t_{n}$ since $s_{n}=t_{n}$ for each $n \in \mathbb{N}$.

Next consider $p_{n+1}(x)$. This will be the vertex $x^{\prime \prime} \in Y_{n+1}$ that is in position $\ell_{n+1}(x)$ since $s_{n+1}=t_{n+1}$. Now $\psi_{n}\left(x^{\prime \prime}\right)$ is the unique vertex in $Y_{n}$ that is in position $\ell_{n+1}(x) \bmod t_{n}$

Since $Y_{n}$ is a single loop, there can only be one vertex in position $\ell_{n+1}(x)$ $\bmod t_{n}$. It follows that $\left(p_{n} \circ \pi_{n}\right)(x)=\left(\psi_{n} \circ p_{n+1}\right)(x)$.

Theorem 3.5. Let $(X, f)$ and $(Y, g)$ be as in Proposition 3.3. Then there exists a continuous, surjective map $p: X \rightarrow Y$ such that $f: X \rightarrow X$ and $g: Y \rightarrow Y$ satisfy that $p \circ f=g \circ p$; that is, the following diagram commutes.

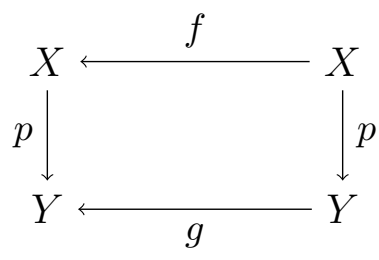

Proof. Since Proposition 3.3 holds for each $n \in \mathbb{N}$, there exists a sequence $\left\{p_{n}\right\}$ of continuous, surjective maps $p_{n}: X_{n} \rightarrow Y_{n}$ satisfying that $p_{n} \circ \pi_{n}=\psi_{n} \circ p_{n+1}$. 
This sequence extends to a continuous, surjective map $p: X \rightarrow Y$ satisfying that $p \circ f=g \circ p$.

\subsection{Semi-Conjugation Between a Finitely Non-Uniquely Ergodic Min- imal Cantor Set and an Adding Machine}

The main objective of this section is to show that there exists a family of finitely non-uniquely ergodic minimal Cantor sets that can be semi-conjugated to an adding machine in such a way that the semi-conjugation map is almost everywhere injective with respect to the uniquely ergodic probability measure associated with a corresponding adding machine. To do this, we will begin by establishing that the following family of combinatorially obtained minimal Cantor sets possesses members that are non-uniquely ergodic.

Proposition 3.6. The minimal Cantor set that is combinatorially obtained from the sequence of winding matrices $\left\{W_{n}\right\}_{n=1}^{\infty}$, with

$$
W_{n}=\left(\begin{array}{ccc}
a_{n} & a_{n} & a_{n} \\
n a_{n}+1 & 2 n a_{n} & 2 \\
n a_{n}+1 & 2 & 2 n a_{n}
\end{array}\right)
$$

is not uniquely ergodic and in fact possesses at least two ergodic invariant probability measures for an appropriately chosen sequence of integers $\left\{a_{n}\right\}_{n=1}^{\infty}$.

Proof. Let

$$
V=\left\{x \in \mathbb{R}^{3}: x_{1}=0, x_{2}, x_{3} \geq 0\right\}
$$

and let $e_{1}, e_{2}, e_{3}$ be the standard basis vectors for $\mathbb{R}^{3}$. The set of vectors $\left\{e_{2}, e_{3}\right\}$ forms a basis for $V$. For any two vectors $x, y \in V$, define

$$
d(x, y)=\arccos \left(\frac{x \cdot y}{\|x\|\|y\|}\right)
$$


that is, $d(x, y)$ gives the angle between the vectors $x$ and $y$. Choose $a_{1}$ large enough so that

$$
d\left(W_{1} e_{2}, W_{1} e_{3}\right)>\frac{\pi}{4}+\frac{1}{2}
$$

$a_{2}$ large enough so that

$$
d\left(W_{1} W_{2} e_{2}, W_{1} W_{2} e_{3}\right)>\frac{\pi}{4}+\frac{1}{3},
$$

and, in general, choose $a_{n}$ large enough so that

$$
d\left(W_{1} \ldots W_{n} e_{2}, W_{1} \ldots W_{n} e_{3}\right)>\frac{\pi}{4}+\frac{1}{n+1}
$$

Since $W_{k} \ldots W_{n}$ serves as a weaker contraction on $V$ than $W_{1} \ldots W_{n}$ for $1<k<n$, if it can be shown that

$$
\lim _{n \rightarrow \infty} d\left(W_{1} \ldots W_{n} e_{2}, W_{1} \ldots W_{n} e_{3}\right)>0
$$

it will follow that

$$
\lim _{n \rightarrow \infty} d\left(W_{k} \ldots W_{n} e_{2}, W_{k} \ldots W_{n} e_{3}\right)>0
$$

for each $k<n$. This will prove that each of the sets $I_{k}=\bigcap_{n=k+1}^{\infty} W_{k} \ldots W_{n} V$ is 2-dimensional, and so $\mathcal{I}=\lim _{\leftarrow} I_{k}$ is 2-dimensional, thus proving that the minimal Cantor set under consideration is not uniquely ergodic and in fact possesses at least two ergodic invariant probability measures. To this end, taking the limit as $n$ approaches infinity of each side of inequality (13) results in

$$
\lim _{n \rightarrow \infty} d\left(W_{1} \ldots W_{n} e_{2}, W_{1} \ldots W_{n} e_{3}\right) \geq \frac{\pi}{4}
$$

and we have the desired result.

The argument above can be generalized to the $m \times m$ case. 
Proposition 3.7. The minimal Cantor set that is combinatorially obtained from the sequence of $m \times m(m \geq 3)$ winding matrices $\left\{W_{n}\right\}_{n=1}^{\infty}$, with

$$
W_{n}=\left(\begin{array}{cccccc}
a_{n} & a_{n} & a_{n} & \ldots & & a_{n} \\
n a_{n}+1 & (m-1) n a_{n} & 2 & 2 & \ldots & 2 \\
n a_{n}+1 & 2 & (m-1) n a_{n} & 0 & \ldots & 0 \\
n a_{n} & 0 & 0 & \ddots & \ddots & \vdots \\
\vdots & & & \ddots & & 0 \\
n a_{n} & 0 & \ldots & & 0 & (m-1) n a_{n}
\end{array}\right)
$$

is not uniquely ergodic and in fact possesses at least $m-1$ ergodic invariant probability measures for an appropriately chosen sequence of integers $\left\{a_{n}\right\}_{n=1}^{\infty}$.

Proof. The proof is analogous to the proof of Proposition 3.6.

For the remainder of this section, let $(X, f)$ with $X=\underset{\pi_{n}}{\lim } X_{n}$ be the minimal Cantor set that is combinatorially obtained as in Proposition 3.7. We will make the additional presumptions that each of the loops of $X_{n}$ possesses the same number of vertices for each $n$ and that any pair of loops in $X_{n}$ has only the splitting vertex in common. Also, let $(Y, g)$ with $Y=\underset{\overleftarrow{\psi_{n}}}{\lim } Y_{n}$ be an adding machine that is combinatorially obtained from the sequence of $1 \times 1$ winding matrices $\left\{((m-1) n+1) a_{n}+2\right\}_{n=1}^{\infty}$ and also satisfies that the single loop of $Y_{1}$ has the same number of vertices as each of the loops of the combinatorial cover $X_{1}$.

Proposition 3.8. For each $n \in \mathbb{N}$ there exist continuous, surjective maps

$$
p_{n}: X_{n} \rightarrow Y_{n} \text { and } p_{n+1}: X_{n+1} \rightarrow Y_{n+1}
$$

satisfying that $p_{n} \circ \pi_{n}=\psi_{n} \circ p_{n+1}$. Moreover, there exists a continuous, surjective map $p: X \rightarrow Y$ satisfying that $p \circ f=g \circ p$.

Proof. Let $t_{n}$ be the number of vertices in $Y_{n}$ and let $s_{n}$ be the number of vertices in each loop of $X_{n}$ for each $n \in \mathbb{N}$. Note that for each $n \in \mathbb{N}$, the number of 
vertices $t_{n+1}$ in the single loop of $Y_{n+1}$ is given by

$$
\left(((m-1) n+1) a_{n}+2\right) t_{n}
$$

and the number of vertices $s_{n+1}$ in each of the loops of $X_{n+1}$ is given by the sum of the corresponding column of $W_{n}$. Since each of the columns of $W_{n}$ sum to $((m-1) n+1) a_{n}+2$, it follows that the number of vertices in each loop of $X_{n+1}$ is given by

$$
\left(((m-1) n+1) a_{n}+2\right) s_{n} .
$$

Moreover, since $s_{1}=t_{1}$, it follows that $s_{n}=t_{n}$ for each $n \in \mathbb{N}$. Hence the hypotheses of Proposition 3.3 and Theorem 3.5 are met and each of the results follows.

It remains to be shown that the semi-conjugation map $p$ of Proposition 3.8 is almost everywhere injective with respect to the uniquely ergodic invariant probability measure associated with the adding machine $(Y, g)$.

For each $n \in \mathbb{N}$, recall that $t_{n}$ represents the number of vertices in the single loop of $Y_{n}$. For each $n \in \mathbb{N} \backslash\{1\}$, define the set

$$
\mathcal{U}_{n}=\left\{y \in Y_{n}: \ell_{n}(y) \in\left\{0,1, \ldots, a_{n-1} t_{n-1}\right\}\right\} .
$$

Note that $\mathcal{U}_{n}$ consists of the first $a_{n-1} t_{n-1}$ vertices of the single loop of $Y_{n}$ for each $n \in\{2,3, \ldots\}$.

Lemma 3.9. Let $y=\left(y_{1}, \ldots, y_{n}, y_{n+1}, \ldots\right) \in Y$ satisfy that for some $n \in \mathbb{N}$, $y_{n+1} \in \mathcal{U}_{n+1}$ and suppose there exist

$$
x=\left(x_{1}, \ldots, x_{n}, x_{n+1}, \ldots\right), x^{\prime}=\left(x_{1}^{\prime}, \ldots, x_{n}^{\prime}, x_{n+1}^{\prime}, \ldots\right) \in X
$$

such that $x, x^{\prime} \in\left\{p^{-1}(y)\right\}$. Then $x_{k}=x_{k}^{\prime}$ for $k=1, \ldots, n$. 
Proof. Suppose $y_{n+1} \in \mathcal{U}_{n+1}$. Then $\ell_{n+1}\left(y_{n+1}\right) \in\left\{0,1, \ldots, a_{n} t_{n}\right\}$. We observe that $\#\left\{p_{n+1}^{-1}\left(y_{n+1}\right)\right\}$ is either 1 or $m$ since each of the $m$ loops of $X_{n+1}$ is projected once around the single loop of $Y_{n+1}$. Moreover, each of $x_{n+1}, x_{n+1}^{\prime} \in\left\{p_{n+1}^{-1}\left(y_{n+1}\right)\right\}$ is located in the same position (though possibly in distinct loops) in $X_{n+1}$ as it is in $Y_{n+1}$ since each of the loops of $X_{n+1}$ are the same length as the single loop of $Y_{n+1}$.

Inspection of the winding matrix corresponding to $\pi_{n}$ (see equation (14)) reveals that each loop of $X_{n+1}$ is wrapped $a_{n}$ times around the first loop of $X_{n}$. Since each loop of $X_{n}$ is of length $t_{n}$, this means that each of the vertices located in a given position chosen from $0,1, \ldots, a_{n} t_{n}$ in $X_{n+1}$ will be mapped to a single vertex in one of the positions $0,1, \ldots, t_{n}-1$ in the first loop of $X_{n}$. In particular, $x_{n+1}, x_{n+1}^{\prime} \in\left\{p_{n+1}^{-1}\left(y_{n+1}\right)\right\}$ are mapped under $\pi_{n}$ to a single vertex in $X_{n}$; that is; $x_{n}=x_{n}^{\prime}$. Now $x_{k}=x_{k}^{\prime}$ for each $k=1, \ldots, n$ by the definition of an inverse limit.

Proposition 3.10. Let $y=\left(y_{1}, \ldots, y_{n}, y_{n+1}, \ldots\right) \in Y$ satisfy that $y_{n} \in \mathcal{U}_{n}$ for infinitely many $n \in \mathbb{N}$. Then $\#\left\{p^{-1}(y)\right\}=1$.

Proof. Let $x, x^{\prime} \in\left\{p^{-1}(y)\right\}$. Since $y_{n} \in \mathcal{U}_{n}$ for infinitely many $n$, Lemma 3.9 yields that there exists an infinite sequence of positive integers $\left\{n_{j}\right\}_{j=1}^{\infty}$ satisfying that $n_{1}<n_{2}<\ldots$ and $x_{k-1}=x_{k-1}^{\prime}$ for $k=1, \ldots, n_{j}$. As an immediate consequence, $x=x^{\prime}$.

We will now show that the set of points $y=\left(y_{1}, \ldots, y_{n}, \ldots\right) \in Y$ satisfying that $y_{n} \in \mathcal{U}_{n}$ for infinitely many $n$ (which, by Proposition 3.10, is exactly the set of points $y \in Y$ which have a unique pre-image under $p$ ) is of full measure with respect to the unique ergodic measure associated with the adding machine $(Y, g)$. This will complete the proof that the semi-conjugation map $p$ is almost everywhere 
injective with respect to this measure.

Proposition 3.11. Let $\mu$ be the unique ergodic invariant probability measure associated with the adding machine $(g, Y)$, let $\mathcal{U}_{n}$ be defined as in equation (15) of page 39, and let

$$
\mathcal{L}_{0}=\left\{y \in Y: y_{n} \in \mathcal{U}_{n} \text { for infinitely many values of } n\right\}
$$

Then $\mu\left(\mathcal{L}_{0}\right)=1$.

Proof. Let

$$
\mathcal{L}_{1}=Y \backslash \mathcal{L}_{0}=\left\{y \in Y: y_{n} \notin \mathcal{U}_{n} \text { for all } n\right\}
$$

We will prove the equivalent statement that $\mu\left(\mathcal{L}_{1}\right)=0$. To this end, let

$$
L=\left\{0, \ldots, t_{1}-1\right\} \times \ldots \times\left\{0, \ldots, t_{n}-1\right\} \times \ldots
$$

where for each $\ell$, the space $\left\{0, \ldots, t_{\ell}-1\right\}$ is equipped with the discrete topology. Note that we can associate to each $y=\left(y_{1}, \ldots, y_{n}, \ldots\right) \in Y$ the corresponding point $\ell_{y}=\left(\ell_{1}\left(y_{1}\right), \ldots, \ell_{n}\left(y_{n}\right), \ldots\right) \in L$ (and vice versa since $Y_{n}$ consists of a single loop for each $n$ ). Hence there is a homeomorphism

$$
H: Y \rightarrow L
$$

Now since $Y_{1}$ consists of $t_{1}$ vertices, $Y_{1}$ carries the probability measure which assigns to each vertex of $Y_{1}$ the weight $\frac{1}{t_{1}}$. For $n=2,3, \ldots$, the combinatorial cover $Y_{n}$ consists of $t_{n}=\left(((m-1)(n-1)+1) a_{n-1}+2\right) t_{n-1}$ vertices, so $Y_{n}$ carries the probability measure $\mu_{n}$ which assigns to each vertex of $Y_{n}$ the weight

$$
\frac{1}{\left(((m-1)(n-1)+1) a_{n-1}+2\right) t_{n-1}} .
$$

Hence $L$ carries the product measure $\tilde{\mu}$ satisfying that $\tilde{\mu}\left(\ell_{y}\right)=\prod_{j=1}^{\infty} \mu_{j}\left(\ell_{j}\left(y_{j}\right)\right)$. 
For each $n \in\{2,3, \ldots\}$, the number of vertices in $Y_{n}$ that are also in $\mathcal{U}_{n}$ is $a_{n-1} t_{n-1}$. The number of vertices in $Y_{n}$ that are not in $\mathcal{U}_{n}$ is given by

$$
t_{n}-a_{n-1} t_{n-1}=\left(((m-1)(n-1)+1) a_{n-1}+2\right) t_{n-1}-a_{n-1} t_{n-1} .
$$

Now

$$
\begin{aligned}
\tilde{\mu}\left(H\left(\mathcal{L}_{1}\right)\right) & =\frac{t_{1}}{t_{1}} \prod_{j=2}^{\infty} \frac{\left(((m-1)(j-1)+1) a_{j-1}+2\right) t_{j-1}-a_{j-1} t_{j-1}}{\left(((m-1)(j-1)+1) a_{j-1}+2\right) t_{j-1}} \\
& =\prod_{j=2}^{\infty}\left(1-\frac{a_{j-1}}{((m-1)(j-1)+1) a_{j-1}+2}\right) .
\end{aligned}
$$

It can be shown that $\lim _{N \rightarrow \infty} \prod_{j=2}^{N}\left(1-\frac{a_{j-1}}{((m-1)(j-1)+1) a_{j-1}+2}\right)=0$. The fact that $H: Y \rightarrow L$ is a homeomorphism and that $Y$ is uniquely ergodic guarantees that $\mu\left(\mathcal{L}_{1}\right)=0$, and we have the desired result.

Together, the results in this section prove the following theorem.

Theorem 3.12. The minimal Cantor set that is combinatorially obtained from the sequence of $m \times m(m \geq 3)$ winding matrices $\left\{W_{n}\right\}_{n=1}^{\infty}$, with

$$
W_{n}=\left(\begin{array}{cccccc}
a_{n} & a_{n} & a_{n} & \ldots & & a_{n} \\
n a_{n}+1 & (m-1) n a_{n} & 2 & 2 & \ldots & 2 \\
n a_{n}+1 & 2 & (m-1) n a_{n} & 0 & \ldots & 0 \\
n a_{n} & 0 & 0 & \ddots & \ddots & \vdots \\
\vdots & & & \ddots & & 0 \\
n a_{n} & 0 & \ldots & & 0 & (m-1) n a_{n}
\end{array}\right)
$$

is finitely non-uniquely ergodic for an appropriately chosen sequence of integers $\left\{a_{n}\right\}_{n=1}^{\infty}$. For such a sequence $\left\{a_{n}\right\}_{n=1}^{\infty}$, this minimal Cantor set is semi-conjugated to an adding machine in such a way that the semi-conjugation map is almost everywhere injective with respect to the unique ergodic probability measure on the adding machine. 


\subsection{Semi-Conjugation Between an Infinitely Non-Uniquely Ergodic Minimal Cantor Set and an Adding Machine}

The main result of the previous section shows that there exists a non-uniquely ergodic minimal Cantor set possessing any finite number of ergodic invariant probability measures we like that is semi-conjugated to an adding machine in such a way that the semi-conjugation is almost everywhere injective with respect to the unique ergodic measure associated with the adding machine. It seems natural that if this can be done with a non-uniquely ergodic minimal Cantor set carrying as many ergodic measures as we like, it should also be possible for a minimal Cantor set carrying an infinite number of ergodic measures. The main result of this section will show that this is indeed the case.

Thus far each of the constructions we have considered have consisted of minimal Cantor sets which can be combinatorially obtained from square winding matrices of the same size. Winding matrices are allowed to be rectangular, however, provided that the size of each winding matrix $W_{n}$ leads to the matrix product $W_{k n}=W_{k} \ldots W_{n}$ being well-defined for each $k<n$. In order to introduce an infinitely non-uniquely ergodic minimal Cantor set, we will consider a particular family of minimal Cantor set that can be combinatorially obtained from certain rectangular winding matrices.

Proposition 3.13. For each $n$, let the $W_{n}$ be given by the $m_{n} \times\left(m_{n}+1\right)$ rectangular matrix

$$
W_{n}=\left(\begin{array}{ccccccc}
a_{n} & a_{n} & & \ldots & & a_{n} & a_{n} \\
\frac{1}{m_{n}-1} n a_{n}+1 & n a_{n} & 2 & 2 & \ldots & 2 & 2 \\
\frac{1}{m_{n}-1} n a_{n}+1 & 2 & n a_{n} & 0 & \ldots & 0 & 0 \\
\frac{1}{m_{n}-1} n a_{n} & 0 & 0 & \ddots & \ddots & \vdots & \vdots \\
\vdots & \vdots & & \ddots & & 0 & 0 \\
\frac{1}{m_{n}-1} n a_{n} & 0 & \ldots & & 0 & n a_{n} & n a_{n}
\end{array}\right)
$$

where $n a_{n}$ is divisible by $m_{n}-1$ and $m_{n}=n+2$ for each $n$. For an appropriately 
chosen sequence of integers $\left\{a_{n}\right\}_{n=1}^{\infty}$, the minimal Cantor set that is combinatorially obtained from the sequence of winding matrices $\left\{W_{n}\right\}_{n=1}^{\infty}$ is not uniquely ergodic and in fact possesses an infinite number of invariant probability measures.

Note that in the proposition above, $W_{1}$ is a $3 \times 4$ matrix, $W_{2}$ is a $4 \times 5$ matrix, $W_{3}$ is a $5 \times 6$ matrix, and so on. In particular, the matrix product $W_{k n}=W_{k} \ldots W_{n}$ is well-defined for each $k<n$.

Proof of Proposition 3.13. For each $n$, let

$$
V^{m_{n}}=\left\{x=\left(x_{1}, \ldots, x_{m_{n}}\right) \in \mathbb{R}^{m_{n}}: x_{1}=0, x_{2}, \ldots, x_{m_{n}} \geq 0\right\}
$$

and let $e_{1}^{\left(m_{n}\right)}, \ldots, e_{m_{n}}^{\left(m_{n}\right)}$ be the standard basis vectors for $\mathbb{R}^{m_{n}}$. The set of vectors $\left\{e_{2}^{\left(m_{n}\right)}, \ldots, e_{m_{n}}^{\left(m_{n}\right)}\right\}$ forms a basis for $V^{m_{n}}$. For any two vectors $x, y \in V^{m_{n}}$, define $d: V^{m_{n}} \rightarrow \mathbb{R}$ to be the angle between the vectors $x$ and $y$ as in equation (12). As in Proposition 3.6, if it can be shown that for any $i \neq j$ with $i, j \neq 1$,

$$
\lim _{n \rightarrow \infty} d\left(W_{1} \ldots W_{n} e_{i}^{\left(m_{n}+1\right)}, W_{1} \ldots W_{n} e_{j}^{\left(m_{n}+1\right)}\right)>0
$$

it will follow that the minimal Cantor set under consideration is not uniquely ergodic. For each $n$, we may choose $a_{n}$ large enough so that

$$
d\left(W_{1} \ldots W_{n} e_{i}^{\left(m_{n}+1\right)}, W_{1} \ldots W_{n} e_{j}^{\left(m_{n}+1\right)}\right)>\frac{\pi}{4}+\frac{1}{n+1}
$$

By taking the limit as $n$ approaches infinity of each side of the above inequality, it can be seen that

$$
\lim _{n \rightarrow \infty} d\left(W_{1} \ldots W_{n} e_{i}^{\left(m_{n}+1\right)}, W_{1} \ldots W_{n} e_{j}^{\left(m_{n}+1\right)}\right) \geq \frac{\pi}{4}
$$

Since this holds for any $i \neq j$ with $i, j \neq 1$, it follows that the set

$$
I_{k}=\bigcap_{n=k+1}^{\infty} W_{k} \ldots W_{n} V^{m_{n}+1}
$$


is at least $\left(m_{k}-1\right)$-dimensional for each $k$. As $\left\{m_{k}\right\}$ is a strictly increasing sequence of positive integers, $\mathcal{I}=\lim _{W_{n}} I_{k}$ must be infinite-dimensional. Hence the minimal Cantor set that is combinatorially obtained from the sequence of winding matrices $\left\{W_{n}\right\}_{n=1}^{\infty}$ is not only non-uniquely ergodic, but in fact possesses an infinite number of ergodic invariant probability measures.

For the remainder of this section, let $(X, f)$ with $X=\underset{\pi_{n}}{\lim } X_{n}$ be the minimal Cantor set that is combinatorially obtained as in Proposition 3.13 and let $(Y, g)$ with $Y=\lim _{\overleftarrow{\psi_{n}}} Y_{n}$ be an adding machine where each single loop $Y_{n}$ has the same number of vertices $t_{n}$ as each of the loops in $X_{n}$; that is, $(Y, g)$ is obtained combinatorially from the sequence of winding matrices $\left\{w_{n}\right\}_{n=1}^{\infty}$ where $w_{n}=\left((n+1) a_{n}+2\right)$ for each $n$, and $X_{1}$ and $Y_{1}$ are both assumed to have $t_{1}$ vertices. We also make the additional presumption that any pair of loops in $X_{n}$ has only the splitting vertex in common.

Lemma 3.14. For each $n \in \mathbb{N}$ there exist continuous, surjective maps $p_{n}: X_{n} \rightarrow Y_{n}$ and $p_{n+1}: X_{n+1} \rightarrow Y_{n+1}$ satisfying that $p_{n} \circ \pi_{n}=\psi_{n} \circ p_{n+1}$. Moreover there exists a continuous, surjective map $p: X \rightarrow Y$ such that $p \circ f=g \circ p$.

Proof. For each $n$, each of the loops of $X_{n}$ has the same number of vertices as the single loop of $Y_{n}$. Since neither Proposition 3.3 nor Theorem 3.5 specify that the winding matrices corresponding to the minimal Cantor set where $X=\underset{\pi_{n}}{\lim } X_{n}$ must be square, both findings hold regardless of the size of these winding matrices, and we have the desired result.

As in equation (15) of page 39 , let

$$
\mathcal{U}_{n}=\left\{y \in Y_{n}: \ell_{n}(y) \in\left\{0,1, \ldots, a_{n-1} t_{n-1}\right\}\right.
$$


Lemma 3.15. Let $y=\left(y_{1}, y_{2}, \ldots, y_{n}, y_{n+1}, \ldots\right) \in Y$ satisfy that for some $n$, $y_{n+1} \in \mathcal{U}_{n+1}$ and suppose there exist

$$
x=\left(x_{1}, \ldots, x_{n}, x_{n+1}, \ldots\right), x^{\prime}=\left(x_{1}^{\prime}, \ldots, x_{n}^{\prime}, x_{n+1}^{\prime}, \ldots\right) \in X
$$

such that $x, x^{\prime} \in\left\{p^{-1}(y)\right\}$. Then $x_{k}=x_{k}^{\prime}$ for $k=1, \ldots, n$.

Proof. Note that the number of loops in $X_{n+1}$ is given by the number of columns in $W_{n}$; since $W_{n}$ is an $m_{n} \times\left(m_{n}+1\right)$ matrix, this number is $m_{n}+1$.

Suppose $y_{n+1} \in \mathcal{U}_{n+1}$. Then $\ell_{n+1}\left(y_{n+1}\right) \in\left\{0,1, \ldots, a_{n} t_{n}\right\}$. We observe that $\#\left\{p_{n+1}^{-1}\left(y_{n+1}\right)\right\}$ is either 1 or $m_{n}+1$ since each of the $m_{n}+1$ loops of $X_{n+1}$ is projected once around the single loop of $Y_{n+1}$. Moreover, each of

$$
x_{n+1}, x_{n+1}^{\prime} \in\left\{p_{n+1}^{-1}\left(y_{n+1}\right)\right\}
$$

is located in the same position (though possibly in distinct loops) in $X_{n+1}$ as it is in $Y_{n+1}$ since each of the loops of $X_{n+1}$ are the same length as the single loop of $Y_{n+1}$.

The remainder of the proof is identical to the second paragraph of the proof of Lemma 3.9.

Proposition 3.16. Let $y=\left(y_{1}, y_{2}, \ldots, y_{n}, y_{n+1}, \ldots\right) \in Y$ satisfy that $y_{n} \in \mathcal{U}_{n}$ for infinitely many $n$. Then $\#\left\{p^{-1}(y)\right\}=1$.

Proof. This is a consequence of Lemma 3.15. The proof is analogous to that of Proposition 3.10 .

We will now show that $p$ is almost everywhere injective with respect to the uniquely ergodic invariant probability measure associated with the adding machine $(Y, g)$. 
Proposition 3.17. Let $\mu$ be the uniquely ergodic invariant probability measure associated with the adding machine $(Y, g)$ and let

$$
\mathcal{L}_{0}=\left\{y \in Y: y_{n} \in \mathcal{U}_{n} \text { for infinitely many values of } n\right\}
$$

Then $\mu\left(\mathcal{L}_{0}\right)=1$.

Proof. As in the proof of Proposition 3.11, we will let

$$
\mathcal{L}_{1}=Y \backslash \mathcal{L}_{0}=\left\{y \in Y: y_{n} \notin \mathcal{U}_{n} \text { for all } n\right\}
$$

and prove the equivalent statement that $\mu\left(\mathcal{L}_{1}\right)=0$. Again, there exists a homeomorphsim $H: Y \rightarrow L$ that assigns to each $y=\left(y_{1}, \ldots, y_{n}, \ldots\right) \in Y$ the corresponding point $\ell_{y}=\left(\ell_{1}\left(y_{1}\right), \ldots, \ell_{n}\left(y_{n}\right), \ldots\right) \in L$, where

$$
L=\left\{0, \ldots, t_{1}-1\right\} \times \ldots \times\left\{0, \ldots, t_{n}-1\right\} \times \ldots
$$

Now $Y_{1}$ carries the probability measure which assigns to each vertex of $Y_{1}$ the weight $\frac{1}{t_{1}}$. For $n=2,3, \ldots$, the combinatorial cover $Y_{n}$ consists of $t_{n}=\left(n a_{n-1}+2\right) t_{n-1}$ vertices, so $Y_{n}$ carries the probability measure $\mu_{n}$ which assigns to each vertex of $Y_{n}$ the weight

$$
\frac{1}{\left(n a_{n-1}+2\right) t_{n-1}} \text {. }
$$

Hence $L$ carries the product measure $\tilde{\mu}$ satisfying that $\tilde{\mu}\left(\ell_{y}\right)=\prod_{j=1}^{\infty} \mu_{j}\left(\ell_{j}\left(y_{j}\right)\right)$.

The number of vertices in $Y_{n}$ that are not in $U_{n}$ for each $n$ is given by

$$
t_{n}-a_{n-1} t_{n-1}=\left(n a_{n-1}+2\right) t_{n-1}-a_{n-1} t_{n-1} .
$$

Now

$$
\begin{aligned}
\tilde{\mu}\left(H\left(\mathcal{L}_{1}\right)\right) & =\frac{t_{1}}{t_{1}} \prod_{j=2}^{\infty} \frac{\left(j a_{j-1}+2\right) t_{j-1}-a_{j-1} t_{j-1}}{\left(j a_{j-1}+2\right) t_{j-1}} \\
& =\prod_{j=2}^{\infty}\left(1-\frac{a_{j-1}}{j a_{j-1}+2}\right) .
\end{aligned}
$$


It can be shown that $\lim _{N \rightarrow \infty} \prod_{j=2}^{N}\left(1-\frac{a_{j-1}}{j a_{j-1}+2}\right)=0$. The homeomorphism $H: Y \rightarrow L$ and the fact that $Y$ is uniquely ergodic guarantees that $\mu\left(\mathcal{L}_{1}\right)=0$, and we have the desired result.

We have proved the following theorem.

Theorem 3.18. The minimal Cantor set that is combinatorially obtained from the sequence of $m_{n} \times\left(m_{n}+1\right)\left(m_{n} \geq 3\right.$ and $\left.m_{n}=n+2\right)$ winding matrices $\left\{W_{n}\right\}_{n=1}^{\infty}$, with

$$
W_{n}=\left(\begin{array}{ccccccc}
a_{n} & a_{n} & & \ldots & & a_{n} & a_{n} \\
\frac{1}{m_{n}-1} n a_{n}+1 & n a_{n} & 2 & 2 & \ldots & 2 & 2 \\
\frac{1}{m_{n}-1} n a_{n}+1 & 2 & n a_{n} & 0 & \ldots & 0 & 0 \\
\frac{1}{m_{n}-1} n a_{n} & 0 & 0 & \ddots & \ddots & \vdots & \vdots \\
\vdots & \vdots & & \ddots & & 0 & 0 \\
\frac{1}{m_{n}-1} n a_{n} & 0 & \ldots & & 0 & n a_{n} & n a_{n}
\end{array}\right),
$$

is infinitely non-uniquely ergodic for an appropriately chosen sequence of integers $\left\{a_{n}\right\}_{n=1}^{\infty}$. For such a sequence $\left\{a_{n}\right\}_{n=1}^{\infty}$, this minimal Cantor set is semi-conjugated to an adding machine in such a way that the semi-conjugation map is almost everywhere injective with respect to the unique ergodic probability measure on the adding machine.

\section{List of References}

[1] P. Collas and D. Klein, "An ergodic adding machine on the Cantor set," L'Enseignement Mathmatique, vol. 40, no. 249, 1994.

[2] J. Gambaudo and M. Martens, "Algebraic topology for minimal Cantor sets," Annales Henri Poincaré, vol. 7, pp. 423-446, 2006.

[3] R. Fokkink and L. Oversteegen, "Homogeneous weak solenoids," Trans. Amer. Math. Soc., vol. 354(9), pp. 3743-3755, 2002. 


\section{CHAPTER 4 \\ Realization Under a Unimodal Map}

\subsection{Introduction}

While the results of Chapter 3 are mathematically interesting in their own right, it is only natural to ask if the non-uniquely ergodic minimal Cantor set of Theorem 3.12 can be realized in a setting more familiar to the dynamical systems community. The main result of this chapter will show that this minimal Cantor set can in fact be taken to be $(\omega(c), q)$, where $q:[0,1] \rightarrow[0,1]$ is a logistic unimodal map with critical point $c$ and $\omega(c)$ is the omega-limit set of this critical point.

\subsection{Kneading Theory and Consequences for Unimodal Maps}

Recall that a unimodal map is a map $u:[0,1] \rightarrow[0,1]$ satisfying $u(0)=u(1)=0$ and for which there exists a critical point $c \in(0,1)$ such that $u$ is increasing to the left of $c$ and decreasing to the right of $c$.

One family of unimodal maps that we will consider is a subset of the family of tent maps as defined in Example 1.2. We will consider those maps $T_{a}:[0,1] \rightarrow[0,1]$ where

$$
T_{a}(x)= \begin{cases}a x, & x \leq \frac{1}{2} \\ a(1-x), & x \geq \frac{1}{2}\end{cases}
$$

and $a \in(0,2]$. Note that each tent map achieves its maximum value at its critical point $x=\frac{1}{2}$.

Closely related to the family of tent maps is the family of stunted tent maps, which consist of those functions $\mathcal{S}_{a, p}:[0,1] \rightarrow[0,1]$ for which the corresponding tent map $T_{a}$ has been "stunted" by replacing its peak with a plateau at a given height $p \in(0,1)$ on a closed, symmetric interval of positive length about $x=\frac{1}{2}$ (see Figure 6). In the case of stunted tent maps, the critical point is taken by 


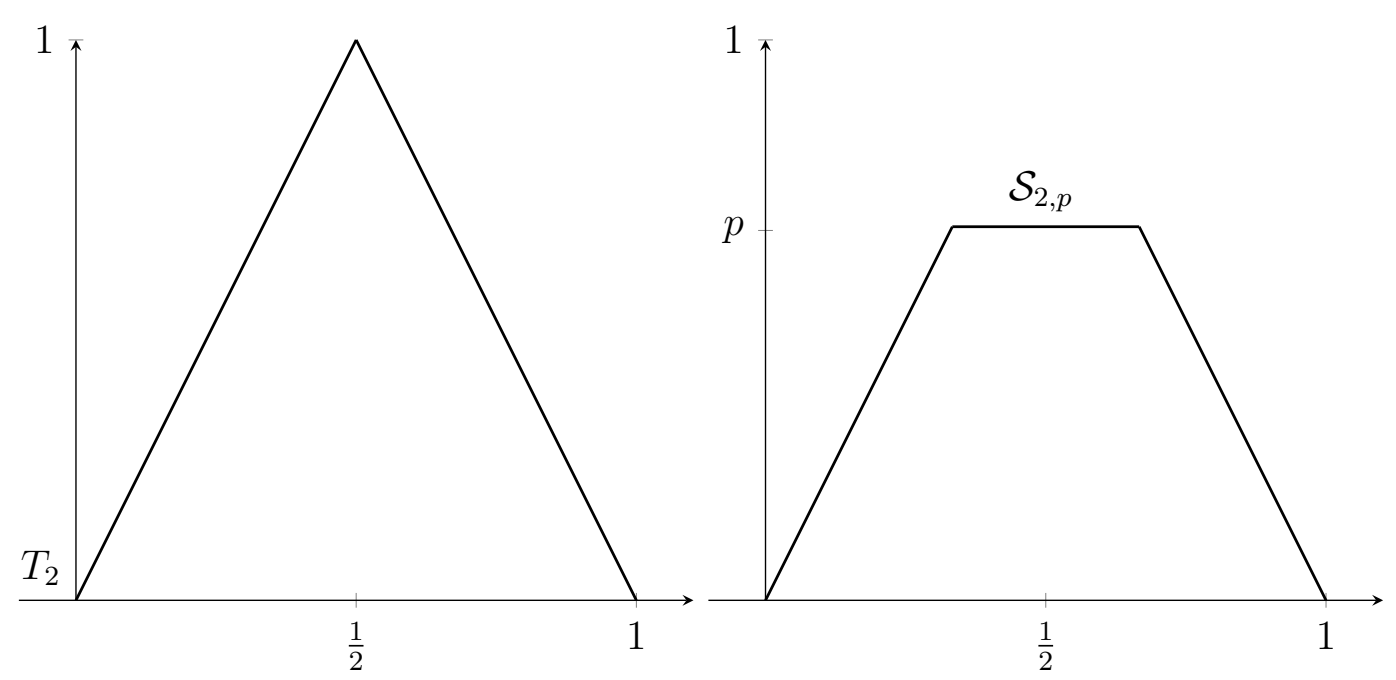

Figure 6. Plots of the tent map $T_{2}$ and a corresponding stunted tent map $\mathcal{S}_{2, p}$. convention to be $x=\frac{1}{2}$.

Another important family of unimodal maps is the family of logistic maps consisting of those functions $q_{a}:[0,1] \rightarrow[0,1]$ defined by $q_{a}(x)=a x(1-x)$ where $a \in(0,4]$ (see Figure 7). Note that in the case of logistic maps, the maximum value occurs at its critical point $x=\frac{1}{2}$ as well.

For a given unimodal map $u$ with critical point $c$, the itinerary of a point $x \in[0,1]$ under $u$ is given by

$$
I(x)=s_{0} s_{1} \ldots \text { with } s_{i}=\left\{\begin{array}{ll}
0 & \text { if } u^{i}(x) \leq c \\
1 & \text { if } u^{i}(x)>c
\end{array} .\right.
$$

Note that if $u^{i}(x)=c$, we take $s_{i}$ to be 0 without loss of generality. The kneading sequence $K$ of a unimodal map is the given by the itinerary of the critical value $u(c)=c_{1}$; that is, $K=I\left(c_{1}\right)$. Moreover, a binary sequence $s \in\{0,1\}^{\mathbb{N}}$ is said to be admissible for $u$ if there exists $x \in[0,1]$ with itinerary $I(x)=s$.

Kneading sequences and kneading theory were introduced and explored by Milnor and Thurston in their paper On Iterated Maps of the Interval (see [1]). 


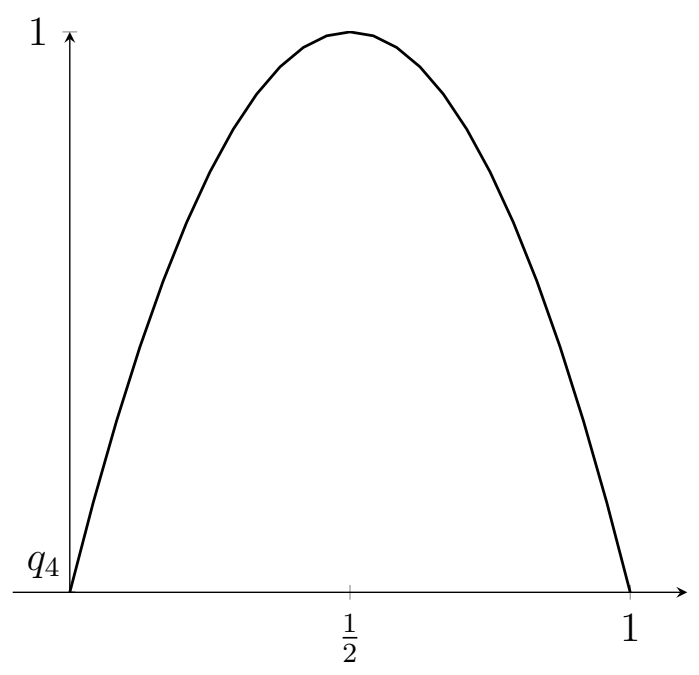

Figure 7. Plot of the logistic unimodal map $q_{4}(x)=4 x(1-x)$.

We will use the related theory developed by Isola and Politi (see [2]) in order to determine whether or not a given binary sequence $s \in\{0,1\}^{\mathbb{N}}$ is admissible as the kneading sequence of a smooth unimodal map.

Let $\sigma:\{0,1\}^{\mathbb{N}} \rightarrow\{0,1\}^{\mathbb{N}}$ be the left shift map defined by

$$
\sigma\left(s_{0} s_{1} \ldots\right)=s_{1} s_{2} \ldots
$$

Then for a unimodal map $u$ and $x \in[0,1]$, the itinerary $I(x)=s_{0} s_{1} s_{2} \ldots$ satisfies that

$$
I(u(x))=s_{1} s_{2} \ldots=\sigma(i(x)) .
$$

Once an ordering that reflects the ordering on the real line has been established, it is known that the admissible binary sequences are exactly those which never become greater than the kneading sequence when shifted.

The method of Isola and Politi begins by associating to a given sequence $s=s_{1} s_{2} \ldots \in\{0,1\}^{\mathbb{N}}$ a number $\tau(s) \in[0,1]$ defined by

$$
\tau=0 . t_{1} t_{2} \ldots=\sum_{k \geq 1} t_{k} 2^{-k}, \text { where } t_{k}=\sum_{i=1}^{k} s_{i}(\bmod 2) .
$$


Let $\sigma:\{0,1\}^{\mathbb{N}} \rightarrow\{0,1\}^{\mathbb{N}}$ be the left shift map, let $T:[0,1] \rightarrow[0,1]$ be the tent map defined by

$$
T(x)=\left\{\begin{array}{ll}
2 x, & x \leq \frac{1}{2} \\
2(1-x), & x \geq \frac{1}{2}
\end{array},\right.
$$

and let $u:[0,1] \rightarrow[0,1]$ be a smooth unimodal map with critical point $c$ and critical value $c_{1}=u(c)$. Direct verification confirms that the following diagram commutes.

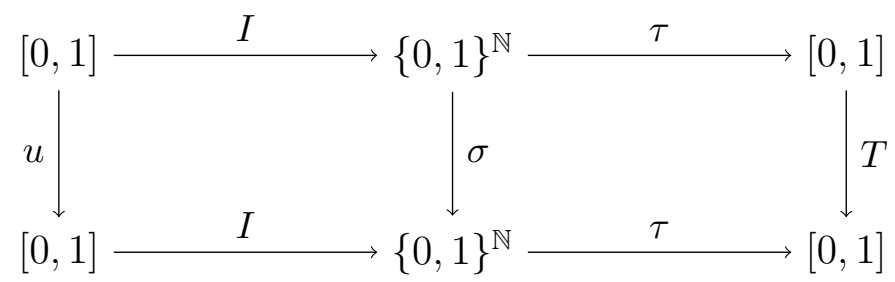

Since $c_{1}$ is the maximum value in the image of $u$ and it can be proved that the map $\tau \circ I$ is increasing (see [3]), the next theorem holds.

Theorem 4.1. (Isola \& Politi) Let $T:[0,1] \rightarrow[0,1]$ be the tent map defined by

$$
T(x)= \begin{cases}2 x, & x \leq \frac{1}{2} \\ 2(1-x), & x \geq \frac{1}{2}\end{cases}
$$

A binary sequence $s \in\{0,1\}^{\mathbb{N}}$ is the kneading sequence of a smooth unimodal map if and only if $\tau:=\tau(s)$ satisfies

$$
T^{k}(\tau) \leq \tau \text { for all } k \geq 0
$$

The following result allows the smooth unimodal map of Theorem 4.1 to be taken to be a logistic map.

Theorem 4.2. (Milnor \& Thurston) Any binary sequence which is admissible as the kneading sequence of a unimodal map can be realized as the kneading sequence of a logistic unimodal map. 
Also related to the kneading sequence of a unimodal map $u$ is the kneading map.

Definition 4.3. Let $J$ be a maximal interval on which $\left.u^{n}\right|_{J}$ is monotone. Then $u^{n}: J \rightarrow u^{n}(J)$ is called a branch. If $c \in \partial J$ (i.e., $c$ is an endpoint of $J$ ), then $u^{n}: J \rightarrow u^{n}(J)$ is called a central branch. If the image of a central branch of $u^{n}$ contains $c$, then $n$ is called a cutting time. The cutting times are denoted by $S_{0}, S_{1}, S_{2}, \ldots$

Definition 4.4. For each $k \in \mathbb{N}$, the difference between two consecutive cutting times $S_{k}$ and $S_{k-1}$ is also a cutting time and the kneading map is defined as

$$
Q(k):=S_{k}-S_{k-1}
$$

Example 4.5. The Feigenbaum unimodal map exhibits cutting times given by powers of 2 ; that is, $S_{0}=1$ and $S_{k}=2 S_{k-1}$ for $k \in \mathbb{N}$. Hence its kneading map is given by

$$
\begin{aligned}
Q(k) & =S_{k}-S_{k-1} \\
& =2 S_{k-1}-S_{k-1} \\
& =S_{k-1}
\end{aligned}
$$

so that $Q(k)=2(k-1)$.

Example 4.6. The Fibonacci unimodal map is named after its cutting times, which are given by the Fibonacci numbers; that is; $S_{0}=1, S_{1}=1$, and $S_{k}=S_{k-1}+S_{k-2}$ for $k \in\{2,3, \ldots\}$. Hence its kneading map is given by

$$
\begin{aligned}
Q(k) & =S_{k}-S_{k-1} \\
& =S_{k-1}+S_{k-2}-S_{k-1} \\
& =S_{k-2}
\end{aligned}
$$

so that $Q(k)=\max \{k-2,0\}$. 
Remark 4.7. It is known that two unimodal maps with the same kneading sequence have the same kneading map, and vice versa.

The proof of the following result can be found in [4].

Theorem 4.8. (Cortez \& Rivera-Letelier) Let u be a smooth unimodal map with critical point c satisfying that $(\omega(c), u)$ is a minimal Cantor set. If $\tilde{u}$ is a unimodal map having the same kneading map as $u$, then $(\omega(\tilde{c}), \tilde{u})$ is also a minimal Cantor set and the space of invariant probability measures of $\tilde{u}$ supported on $\omega(\tilde{c})$ is homeomorphic to that of $u$.

In particular, the above states that the number of ergodic measures of $u$ supported on $\omega(c)$ is the same as those of $\tilde{u}$ supported on $\omega(\tilde{c})$.

\subsection{Obtaining the Desired Unimodal Map}

This section will culminate in the main result proving the existence of a logistic unimodal map $q$ for which $(\omega(c), q)$ is non-uniquely ergodic and may be semiconjugated to an adding machine in such a way that the semi-conjugation map is almost everywhere injective.

To prove this result, we will begin by showing that there exists a minimal Cantor set under the tent map $T$ of Theorem 4.1 that is homeomorphic to a non-uniquely ergodic combinatorially obtained minimal Cantor set satisfying the hypotheses of Theorem 3.12 .

Remark 4.9. By taking $m=3$ in Theorem 3.12 , it can be said that the minimal Cantor set $(X, f)$ with $X=\underset{\pi_{n}}{\lim } X_{n}$ that is combinatorially obtained from the sequence of winding matrices $\left\{W_{n}\right\}_{n=1}^{\infty}$, where

$$
W_{n}=\left(\begin{array}{ccc}
a_{n} & a_{n} & a_{n} \\
n a_{n}+1 & 2 n a_{n} & 2 \\
n a_{n}+1 & 2 & 2 n a_{n}
\end{array}\right)
$$


is non-uniquely ergodic for an appropriately chosen sequence of integers $\left\{a_{n}\right\}_{n=1}^{\infty}$. For such a sequence $\left\{a_{n}\right\}_{n=1}^{\infty}$, this minimal Cantor set is semi-conjugated to the adding machine $(Y, g)$ with $Y=\lim _{\psi_{n}} Y_{n}$ satisfying that, for each $n$, the single loop $Y_{n}$ has the same number of vertices $t_{n}$ as each of the three loops in $X_{n}$. Moreover, the semi-conjugation map $p$ is almost everywhere injective with respect to the unique ergodic probability measure $\mu$ on $(Y, g)$.

Definition 4.10. Given a finite set of elements $\mathcal{W}$, a word $\omega$ of length $N$ in $\mathcal{W}$ is given by $\omega=\omega_{1} \ldots \omega_{N}$ where $\omega_{i}$ is a single element of $\mathcal{W}$ for each $i \in\{1, \ldots N\}$. The set of all such words it denoted by $\mathcal{W}^{N}$.

Lemma 4.11. Fix $N \in\{2,3, \ldots\}$. Let $I_{0}, I_{1}$, and $I_{2}$ be compact, pairwise disjoint subsets of $[0,1]$ and suppose that $\mathfrak{o}:[0,1] \rightarrow[0,1]$ is a continuous, piecewise strictly monotone map with monotone and onto branches $\mathfrak{o}_{k}: I_{k} \rightarrow[0,1]$ for $k=0,1,2$ (see Figure 8). Then for all words $\omega=\omega_{1} \omega_{2} \ldots \omega_{N-1} \in\{0,1,2\}^{N-1}$ there exists a unique, compact interval $I^{\omega} \subset I_{0}$ such that

1. $\mathfrak{o}^{\ell}\left(I^{\omega}\right) \subseteq I_{\omega_{\ell}}$ for $\ell=1, \ldots, N-1$ with equality only in the case when $\ell=N-1$.

2. $\mathfrak{o}^{N}: I^{\omega} \rightarrow[0,1]$ is monotone and onto.

Proof. The proof is by induction on $N$. For the base case, let $N=2$. Then $\omega=\omega_{1}$ where $\omega_{1} \in\{0,1,2\}$. Since $\mathfrak{o}_{0}\left(I_{0}\right)=[0,1]$, there exists a unique, compact interval given by $\mathfrak{o}_{0}^{-1}\left(I_{k}\right) \subset I_{0}$ for each $k \in\{0,1,2\}$. Set $I^{\omega_{1}}=\mathfrak{o}_{0}^{-1}\left(I_{\omega_{1}}\right)$. Then $\mathfrak{o}\left(I^{\omega_{1}}\right)=I_{\omega_{1}}$. Moreover, $\mathfrak{o}^{2}: I^{\omega_{1}} \rightarrow[0,1]$ is monotone and onto. Monotonicity follows from the fact that $\left.\mathfrak{o}\right|_{I_{0}}$ is monotone and surjectivity follows from the fact that $\mathfrak{o}^{2}\left(I^{\omega_{1}}\right)=[0,1]$.

For the induction hypothesis, suppose that for each word

$$
\tilde{\omega}=\omega_{1} \ldots \omega_{N-1} \in\{0,1,2\}^{N-1}
$$




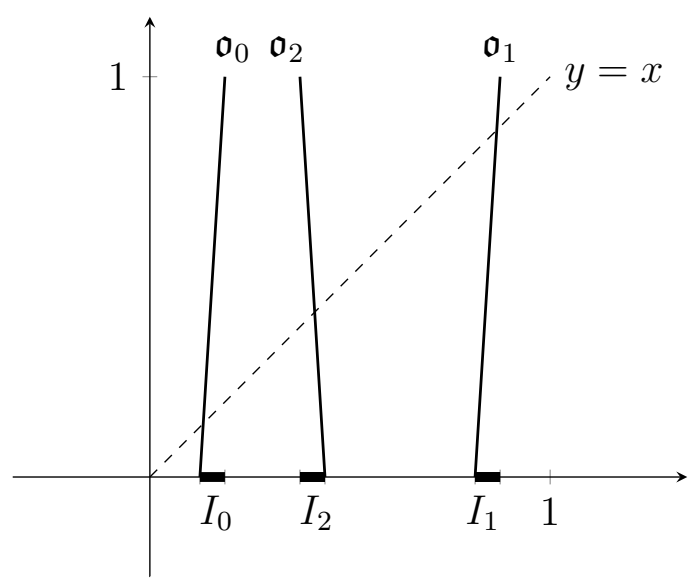

Figure 8. A possible configuration of the intervals $I_{k}$ and the branches $\mathfrak{o}_{k}: I_{k} \rightarrow[0,1](k=0,1,2)$ of the map $\mathfrak{o}$ as described in Lemma 4.11.

there exists a unique, compact interval $I^{\tilde{\omega}} \subset I_{0}$ such that

1. $\mathfrak{o}^{\ell}\left(I^{\tilde{\omega}}\right) \subseteq I_{\omega_{\ell}}$ for $\ell=1, \ldots, N-1$ with equality only in the case when $\ell=N-1$.

2. $\mathfrak{o}^{N}: I^{\tilde{\omega}} \rightarrow[0,1]$ is monotone and onto.

We will show that the result holds for each word $\omega \in\{0,1,2\}^{N}$. For such a word, $\omega=\tilde{\omega} \omega_{N}$ where $\tilde{\omega} \in\{0,1,2\}^{N-1}$. Set $I^{\omega}=\left(\left.\mathfrak{o}^{N}\right|_{I^{\tilde{\omega}}}\right)^{-1}\left(I_{\omega_{N}}\right)$. Then $I^{\omega} \subset I^{\tilde{\omega}} \subset I_{0}$ and $\mathfrak{o}^{\ell}\left(I^{\omega}\right) \subset I_{\omega_{\ell}}$ for $\ell=1, \ldots, N-1$ by the induction hypothesis. Moreover, $\mathfrak{o}^{N}\left(I^{\omega}\right)=I_{\omega_{N}}$ by direct computation. Finally, $\mathfrak{o}^{N+1}: I^{\omega} \rightarrow[0,1]$ is monotone and onto since $\left.\mathfrak{o} \circ \mathfrak{o}^{N}\right|_{I^{\omega}}$ is monotone and $\mathfrak{o}^{N+1}\left(I^{\omega}\right)=[0,1]$.

Lemma 4.12. Fix $N \in\{2,3, \ldots\}$ and let $\omega, \omega^{\prime} \in\{0,1,2\}^{N-1}$. Let $I_{0}, I_{1}, I_{2}$, $\mathfrak{o}:[0,1] \rightarrow[0,1]$, and $\mathfrak{o}_{k}: I_{k} \rightarrow[0,1]$ for $k=0,1,2$ be as in Lemma 4.11. Suppose that $I_{0}$ satisfies that $\mathfrak{o}^{\ell}\left(\partial I_{0}\right) \cap \operatorname{int}\left(I_{0}\right)=\varnothing$ for all $\ell \in \mathbb{N}$ and let $I^{\omega}, I^{\omega^{\prime}} \subset I_{0}$ be pairwise distinct intervals satisfying that $\mathfrak{o}^{N-1}\left(I^{\omega}\right)=\mathfrak{o}^{N-1}\left(I^{\omega^{\prime}}\right)=I_{0}$ (note that this can be done by Lemma 4.11). Then

$$
\mathfrak{o}^{\ell_{1}}\left(I^{\omega}\right) \cap \mathfrak{o}^{\ell_{2}}\left(I^{\omega^{\prime}}\right) \neq \varnothing \text { for some } \ell_{1} \leq \ell_{2} \in \mathbb{N} \Longrightarrow \mathfrak{o}^{\ell_{1}}\left(I^{\omega}\right)=\mathfrak{o}^{\ell_{2}}\left(I^{\omega^{\prime}}\right) .
$$


Proof. For the sake of contradiction, suppose that $\mathfrak{o}^{\ell_{1}}\left(I^{\omega}\right) \cap \mathfrak{o}^{\ell_{2}}\left(I^{\omega^{\prime}}\right) \neq \varnothing$ and that $\mathfrak{o}^{\ell_{1}}\left(I^{\omega}\right) \neq \mathfrak{o}^{\ell_{2}}\left(I^{\omega^{\prime}}\right)$. Then there are three possibilities: either $\mathfrak{o}^{\ell_{2}}\left(I^{\omega^{\prime}}\right) \subset \operatorname{int}\left(\mathfrak{o}^{\ell_{1}}\left(I^{\omega}\right)\right)$, $\partial \mathfrak{o}^{\ell_{1}}\left(I^{\omega}\right) \cap \operatorname{int}\left(\mathfrak{o}^{\ell_{2}}\left(I^{\omega^{\prime}}\right)\right) \neq \varnothing$, or $\mathfrak{o}^{\ell_{1}}\left(I^{\omega}\right)$ and $\mathfrak{o}^{\ell_{2}}\left(I^{\omega^{\prime}}\right)$ intersect at a common endpoint only.

Case 1: Suppose $\mathfrak{o}^{\ell_{2}}\left(I^{\omega^{\prime}}\right) \subset \operatorname{int}\left(\mathfrak{o}^{\ell_{1}}\left(I^{\omega}\right)\right)$. Then

$$
I_{0}=\mathfrak{o}^{N-1-\ell_{2}}\left(\mathfrak{o}^{\ell_{2}}\left(I^{\omega^{\prime}}\right)\right) \subset \mathfrak{o}^{N-1-\ell_{2}}\left(\mathfrak{o}^{\ell_{1}}\left(I^{\omega}\right)\right) \subset I_{k}
$$

for some $k \in\{0,1,2\}$, an immediate contradiction.

Case 2: If $\partial \mathfrak{o}^{\ell_{1}}\left(I^{\omega}\right) \cap \operatorname{int}\left(\mathfrak{o}^{\ell_{2}}\left(I^{\omega^{\prime}}\right)\right) \neq \varnothing$, then there exists an endpoint $d$ of $\mathfrak{o}^{\ell_{1}}\left(I^{\omega}\right)$ that also lies in the interior of $\mathfrak{o}^{\ell_{2}}\left(I^{\omega^{\prime}}\right)$. Since $d$ is an endpoint of $\mathfrak{o}^{\ell_{1}}\left(I^{\omega}\right)$, $d^{\prime}=\mathfrak{o}^{N-1-\ell_{1}}(d)$ is an endpoint of $I_{0}$ by monotonicity. Since $d$ is also an element of the interior of $\mathfrak{o}^{\ell_{2}}\left(I^{\omega^{\prime}}\right), d^{\prime}=\mathfrak{o}^{N-1-\ell_{2}}(d)$ as well and is in the interior of $\mathfrak{o}^{N-1-\ell_{2}}\left(\operatorname{int}\left(\mathfrak{o}^{\ell_{1}}\left(I^{\omega}\right)\right)\right)$. Hence $\mathfrak{o}^{\ell_{2}-\ell_{1}}\left(d^{\prime}\right)$ must be in the interior of $I_{0}$. Now an iterate of $d^{\prime}$, which is itself an endpoint of $I_{0}$, intersects the interior of $I_{0}$. This contradicts the assumption that $\mathfrak{o}^{\ell}\left(\partial I_{0}\right) \cap \operatorname{int}\left(I_{0}\right)=\varnothing$ for all $\ell \in \mathbb{N}$.

Case 3: If $\mathfrak{o}^{\ell_{1}}\left(I^{\omega}\right)$ and $\mathfrak{o}^{\ell_{2}}\left(I^{\omega^{\prime}}\right)$ intersect only at a common endpoint, monotonicity implies that not both $\mathfrak{o}^{N-1-\ell_{1}}\left(\mathfrak{o}^{\ell_{1}}\left(I^{\omega}\right)\right), \mathfrak{o}^{N-1-\ell_{2}}\left(\mathfrak{o}^{\ell_{2}}\left(I^{\omega}\right)\right)$ can be $I_{0}$, a contradiction.

For the remainder of this section, let $(X, f)$ with $X=\underset{\pi_{n}}{\lim } X_{n}$ and $(Y, g)$ with $Y=\lim _{\overleftarrow{\psi_{n}}} Y_{n}$ be the minimal Cantor sets defined as in Remark 4.9. Then $t_{n}$ is the number of vertices in the single loop of $Y_{n}$ as well as the number of vertices in each loop of $X_{n}$. Inspection of the sequence of winding matrices associated with the sequence of combinatorial covers $\left\{\pi_{n}\right\}_{n=1}^{\infty}$ given in Remark 4.9 reveals that $t_{n+1}=\left((2 n+1) a_{n}+2\right) t_{n}$ for each $n \in \mathbb{N}$.

Let $T:[0,1] \rightarrow[0,1]$ be the tent map defined in Theorem 4.1 and suppose that for each $n \in \mathbb{N} \cup\{0\}$, some iterate of $T$ is monotone and onto on each of three 
compact, pairwise disjoint intervals $I_{0}^{n}, I_{1}^{n}, I_{2}^{n}$ (note that this can be done as $T$ is a piecewise monotone map). Lemma 4.11 implies that there exist unique, pairwise disjoint intervals $I_{0}^{n+1}, I_{1}^{n+1}, I_{2}^{n+1} \subset I_{0}^{n}$ satisfying that

$$
\begin{aligned}
& \text { - } I_{0}^{n+1} \mapsto T\left(I_{0}^{n+1}\right) \mapsto \ldots \mapsto T^{t_{n}-1}\left(I_{0}^{n+1}\right) \mapsto T^{t_{n}-1}\left(I_{0}^{n+1}\right)=I_{0}^{n} \\
& \text { - } I_{1}^{n+1} \mapsto T\left(I_{1}^{n+1}\right) \mapsto \ldots \mapsto T^{t_{n}-1}\left(I_{1}^{n+1}\right) \mapsto T^{t_{n}-1}\left(I_{0}^{n+1}\right)=I_{0}^{n} \\
& \text { - } I_{2}^{n+1} \mapsto T\left(I_{2}^{n+1}\right) \mapsto \ldots \mapsto T^{t_{n}-1}\left(I_{2}^{n+1}\right) \mapsto T^{t_{n}-1}\left(I_{2}^{n+1}\right)=I_{0}^{n}
\end{aligned}
$$

and $T^{\ell}\left(I_{k}^{n+1}\right)$ is a proper subset of one of $I_{0}^{n}, I_{1}^{n}, I_{2}^{n}$ for $\ell=1, \ldots, t_{n}-1$ and $k \in\{0,1,2\}$. Lemma 4.12 additionally allows us to presume that if $T^{\ell_{1}}\left(I_{k_{1}}^{n}\right) \cap T^{\ell_{2}}\left(I_{k_{2}}^{n}\right) \neq \varnothing$, then $T^{\ell_{1}}\left(I_{k_{1}}^{n}\right)=T^{\ell_{2}}\left(I_{k_{2}}^{n}\right)$. Moreover, Lemma 4.11 results in a natural inclusion of the orbit of $I_{k}^{n+1}$ for each $k$ within the orbits of $I_{j}^{n}$ for $j=0,1,2$. We can specifically assume that this inclusion reflects the winding matrices $\left\{W_{n}\right\}_{n=1}^{\infty}$ as defined in Remark 4.9.

For each $n \in \mathbb{N}$ and $k \in\{0,1,2\}$, the intervals $I_{k}^{n+1}$ are pairwise disjoint with orbits under $T$ that come to coincide at some iterate $t$, where $1 \leq t \leq t_{n}-1$. The orbits of these intervals can thus be thought of in the sense of a combinatorial cover, as will be explained in detail within the proof of the next proposition.

Proposition 4.13. For each $n \in \mathbb{N}$ and each $k \in\{0,1,2\}$ define

$$
\mathcal{O}_{n}=\left\{\bigcup_{k=0}^{2} T^{t}\left(I_{k}^{n}\right): 0 \leq t \leq t_{n}-1\right\} .
$$

Then there exists a combinatorial cover $X_{n}^{\mathcal{O}}$ and a homeomorphism $h_{n}: \mathcal{O}_{n} \rightarrow X_{n}^{\mathcal{O}}$ for each $n \in \mathbb{N}$.

Proof. For each $k \in\{0,1,2\}$ and $n \in \mathbb{N}$, consider the subset of the orbit of $I_{k}^{n}$ under the tent map $T$ given by

$$
\lambda\left(I_{k}^{n}\right)=\left\{I_{k}^{n}, T\left(I_{k}^{n}\right), \ldots, T^{t_{n}-1}\left(I_{k}^{n}\right)\right\} .
$$


By definition, $\lambda\left(I_{k}^{n}\right)$ consists of exactly $t_{n}$ intervals for each $k$ and $n$.

To form the combinatorial cover $X_{n}^{\mathcal{O}}$, begin by creating a vertex for each interval in $\lambda\left(I_{0}^{n}\right)$. Place a directed edge between two vertices $x$ and $y$ if and only if the interval $T^{\ell}\left(I_{0}^{n}\right)$ associated with the vertex $x$ satisfies that $T^{\ell+1}\left(I_{0}^{n}\right)$ is the interval associated with the vertex $y$. Proceed in the same fashion with $\lambda\left(I_{1}^{n}\right)$ and $\lambda\left(I_{2}^{n}\right)$, but if any of the intervals in either of these is the same as an interval $I$ in $I_{0}^{n}$, associate the interval with the same vertex that $I$ was associated with. Note that this construction results in the vertex associated with $I_{0}^{n-1}$ being the splitting vertex of $X_{n}^{\mathcal{O}}$ and the intervals $T\left(I_{0}^{n}\right), T\left(I_{1}^{n}\right)$, and $T\left(I_{2}^{n}\right)$ corresponding to the vertices that are the images of this splitting vertex.

This construction of $X_{n}^{\mathcal{O}}$ results in a natural homeomorphism $h_{n}: \mathcal{O}_{n} \rightarrow X_{n}^{\mathcal{O}}$ for each $n$.

As we wish to consider minimal Cantor sets, the next step is to the prove that there also exists a sequence of combinatorial refinements $\left\{\pi_{n}^{\mathcal{O}}: X_{n+1}^{\mathcal{O}} \rightarrow X_{n}^{\mathcal{O}}\right\}_{n=1}^{\infty}$.

Proposition 4.14. For each $n$, there exists a combinatorially refinement

$$
\pi_{n}^{\mathcal{O}}: X_{n+1}^{\mathcal{O}} \rightarrow X_{n}^{\mathcal{O}}
$$

that can be described by the sequence of winding matrices associated with the combinatorial refinements $\pi_{n}: X_{n+1} \rightarrow X_{n}$.

Proof. For each $n \in \mathbb{N}, X_{n}^{\mathcal{O}}$ consists of three loops of length

$$
t_{n}=\left((2(n-1)+1) a_{n-1}+2\right) t_{n-1} .
$$

Due to how the sets $\mathcal{O}_{n}$ were chosen, a combinatorial refinement $\pi_{n}^{\mathcal{O}}: X_{n+1}^{\mathcal{O}} \rightarrow X_{n}^{\mathcal{O}}$ may be defined in such a way that the corresponding sequence of winding matrices is given by $\left\{W_{n}\right\}_{n=1}^{\infty}$ (the same sequence used to obtained the non-uniquely ergodic minimal Cantor set $(X, f)$ of Remark 4.9). 
This sequence of combinatorial refinements together with the sequence of homeomorphisms from Proposition 4.13 allows us to project between the sets $\mathcal{O}_{n}$.

Proposition 4.15. For each $n \in \mathbb{N}$, there exists a continuous, surjective map $\psi_{n}^{\mathcal{O}}: \mathcal{O}_{n+1} \rightarrow \mathcal{O}_{n}$ which may be described by the winding matrices $\left\{W_{n}\right\}_{n=1}^{\infty}$ of Remark 4.9.

Proof. Lemmas 4.13 and 4.14 yield the following diagram.

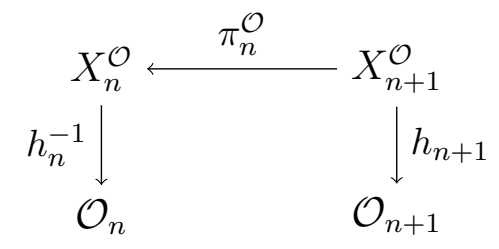

This diagram implies the existence of a continuous, surjective map $\psi_{n}^{\mathcal{O}}: \mathcal{O}_{n+1} \rightarrow \mathcal{O}_{n}$ given by $\psi_{n}^{\mathcal{O}}=h_{n}^{-1} \circ \pi_{n}^{\mathcal{O}} \circ h_{n+1}$ for each $n \in \mathbb{N}$ which may be described by the winding matrices $\left\{W_{n}\right\}_{n=1}^{\infty}$ associated with $\left\{\pi_{n}^{\mathcal{O}}\right\}_{n=1}^{\infty}$.

We now have two inverse limit sequences $\left\{X_{n}^{\mathcal{O}}, \pi_{n}^{\mathcal{O}}\right\}_{n=1}^{\infty}$ and $\left\{\mathcal{O}_{n}, \psi_{n}^{\mathcal{O}}\right\}_{n=1}^{\infty}$ and will show that each can be used to obtain a minimal Cantor set.

Proposition 4.16. Let $\left(X^{\mathcal{O}}, f^{\mathcal{O}}\right)$ be the minimal Cantor set combinatorially obtained from $X^{\mathcal{O}}=\lim _{\overleftarrow{\pi_{n}^{\mathcal{O}}}} X_{n}^{\mathcal{O}}$ and let $\mathcal{O}=\lim _{\overleftarrow{\psi_{n}^{\mathcal{O}}}} \mathcal{O}_{n}$. Then $X^{\mathcal{O}}$ is homeomorphic to $\mathcal{O}$. Moreover, $(\mathcal{O}, T)$ is a minimal Cantor set that is topologically conjugate to $\left(X^{\mathcal{O}}, f^{\mathcal{O}}\right)$.

Proof. Since Lemmas 4.13 and 4.15 hold for each $n \in \mathbb{N}$, there exists a sequence $\left\{\tilde{h}_{n}: \mathcal{O}_{n} \rightarrow X_{n}^{\mathcal{O}}\right\}_{n=1}^{\infty}$ of homeomorphisms satisfying that $\tilde{h}_{n} \circ \pi_{n}^{\mathcal{O}}=\psi_{n}^{\mathcal{O}} \circ \tilde{h}_{n+1}$. This sequence extends to a homeomorphism $h: \mathcal{O} \rightarrow X^{\mathcal{O}}$. Now $h \circ f^{\mathcal{O}}=T \circ h$, since $T$ is the map that describes the orbits of $I_{k}^{n}$ for each $n$ and hence also the orbits of $\mathcal{O}$. Now, $\mathcal{O}$ is topologically conjugate to $X^{\mathcal{O}}$ and $(\mathcal{O}, T)$ must also be a minimal Cantor set. 
The above specifically proves that there exists a minimal Cantor set under the tent map $T$ that is homeomorphic to the minimal Cantor set $\left(X^{\mathcal{O}}, f^{\mathcal{O}}\right)$ which was combinatorially obtained in a similar fashion to the non-uniquely ergodic minimal Cantor set $(X, f)$ of Remark 4.9. Since $(X, f)$ is semi-conjugated to the adding machine $(Y, g)$ of Remark 4.9, our next step is to show that $\left(X^{\mathcal{O}}, f^{\mathcal{O}}\right)$ (and hence $(\mathcal{O}, T))$ can be semi-conjugated to $(X, f)$.

Proposition 4.17. There exists a continuous, surjective map $p^{\mathcal{O}}: X \rightarrow X^{\mathcal{O}}$ satisfying that $p^{\mathcal{O}} \circ f=f^{\mathcal{O}} \circ p^{\mathcal{O}}$.

Proof. Each of the three loops of the combinatorial cover $X_{n}$ consists of $t_{n}$ vertices with the only common vertex being the splitting vertex. Each of the three loops of the combinatorial cover $X_{n}^{\mathcal{O}}$ also consists of $t_{n}$ vertices, but in this case each loop may have multiple vertices in common.

Let $U_{1}, U_{2}$, and $U_{3}$ be the images of the splitting vertex in $X_{n}$ and let $U_{1}^{\mathcal{O}}, U_{2}^{\mathcal{O}}$, and $U_{3}^{\mathcal{O}}$ be the images of the splitting vertex in $X_{n}^{\mathcal{O}}$. Define the $p_{n}^{\mathcal{O}}: X_{n} \rightarrow X_{n}^{\mathcal{O}}$ so that the vertex $x \in \lambda\left(U_{i}\right)$ that is located in position $\ell_{n}(x)$ in $X_{n}$ is mapped to the vertex $y \in \lambda\left(U_{i}^{\mathcal{O}}\right)$ located in position $\ell_{n}$ in $X_{n}^{\mathcal{O}}$. By definition, $p_{n}^{\mathcal{O}}$ is continuous and onto for each $n$ and satisfies that $p_{n}^{\mathcal{O}} \circ \pi_{n}=\pi_{n}^{\mathcal{O}} \circ p_{n+1}^{\mathcal{O}}$.

The sequence of maps $\left\{p_{n}: X_{n} \rightarrow X_{n}^{\mathcal{O}}\right\}_{n=1}^{\infty}$ may then be extended to a continuous, surjective map $p^{\mathcal{O}}: X \rightarrow X^{\mathcal{O}}$ satisfying that $p^{\mathcal{O}} \circ f=f^{\mathcal{O}} \circ p^{\mathcal{O}}$.

Proposition 4.18. There exists a continuous, surjective map $\rho: X^{\mathcal{O}} \rightarrow Y$ satisfying that $\rho \circ f^{\mathcal{O}}=g \circ \rho$.

Proof. Since each of the loops of $X_{n}^{\mathcal{O}}$ has the same number of vertices as the single loop of $Y_{n}$ for $n=0,1, \ldots$, Theorem 3.5 guarantees the existence of a continuous, surjective map $\rho: X^{\mathcal{O}} \rightarrow Y$ with the desired property.

We have the following commutative diagram. 


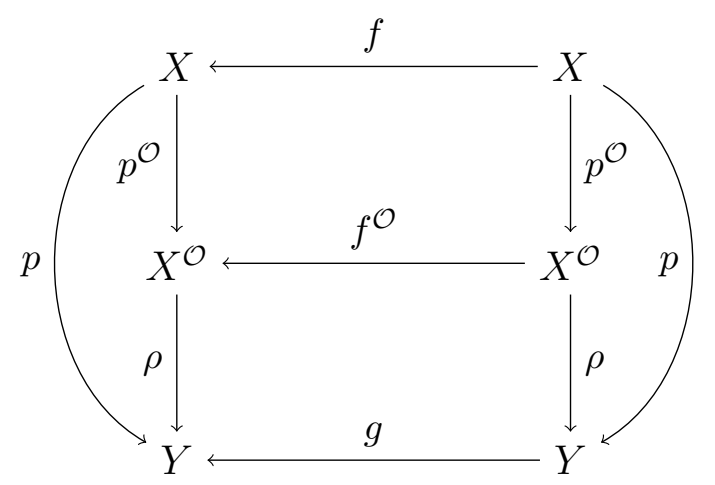

Now $\left(X^{\mathcal{O}}, f^{\mathcal{O}}\right)$ (and hence $(\mathcal{O}, T)$ ) is semi-conjugated to the adding machine $(Y, g)$. It remains to be shown that $\rho$ is in fact almost everywhere injective with respect to the unique ergodic invariant probability measure on $(Y, g)$.

Proposition 4.19. The map $\rho: X^{\mathcal{O}} \rightarrow Y$ is almost everywhere injective with respect to the unique ergodic measure $\mu$ on $(Y, g)$.

Proof. Since the diagram

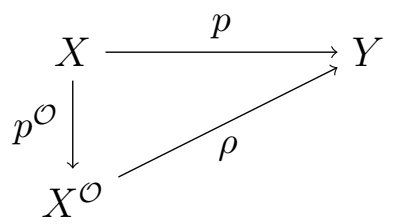

commutes, $\left\{y \in Y: \# \rho^{-1}(y) \geq 2\right\} \subseteq\left\{y \in Y: \# p^{-1}(y) \geq 2\right\}$. Moreover, $\left.\mu\left(Y: \# p^{-1}(y) \geq 2\right\}\right)=0$ as a consequence of Proposition 3.11, and hence $\mu\left(\left\{y \in Y: \# \rho^{-1}(y) \geq 2\right\}\right)=0$ as well.

We have proved the following result.

Theorem 4.20. There exists a non-uniquely ergodic minimal Cantor set under the tent map $T$ of Theorem 4.1 that can be semi-conjugated to a specific adding machine in such a manner that the semi-conjugation map is almost everywhere injective with respect to the single ergodic invariant probability measure associated with the adding machine. 
This theorem brings us one step close to our ultimate goal of proving that the minimal Cantor set of Remark 4.9 can be taken to be $(\omega(c), q)$ for some logistic map $q$ with critical point $c$. However, $\mathcal{O}$ is not the omega limit set of the critical point of $T$. We can make it the omega limit set of the critical point of a particular corresponding stunted tent map, however. To this end, let $\nu$ be the maximal element of the Cantor set $\mathcal{O}$ and let $\mathcal{S}$ be the stunted tent map corresponding to $T$ with plateau at height $\nu$. Now $(\omega(\nu), \mathcal{S})$ is exactly the same as $(\mathcal{O}, T)$. Moreover, $\nu$ is the critical value of $\mathcal{S}$ and we can consider the kneading sequence $K=I(\nu)$ of $\mathcal{S}$.

Proposition 4.21. The binary sequence $K=I(\nu)$ is admissible as the kneading sequence of a logistic unimodal map.

Proof. The tent map $T$ and the stunted tent map $\mathcal{S}$ agree on $\mathcal{O}$. Since $\nu$ is the maximal element of the Cantor set $\mathcal{O}$ and $T$ maps $\mathcal{O}$ to itself, $T^{k}(\nu) \leq \nu$ for all $k \in \mathbb{N} \cup\{0\}$. It immediately follows from Theorem 4.1 and Theorem 4.2 that there exists a quadratic unimodal map $q$ with kneading sequence given by $K$.

Proposition 4.22. Let $q:[0,1] \rightarrow[0,1]$ be the logistic unimodal map whose existence was guaranteed by Proposition 4.21. The minimal Cantor set $(\omega(c), q)$ is non-uniquely ergodic.

Proof. Since two unimodal maps with the same kneading invariant also have the same kneading map (see Remark 4.7), it follows immediately from Theorem 4.8 and the previous results in this section that $(\omega(c), q)$ is non-uniquely ergodic.

Together, the results of this section prove the subsequent result.

Theorem 4.23. There exists a logistic unimodal map with critical point $c$ satisfying that $\omega(c)$ is a non-uniquely ergodic minimal Cantor set that can be semiconjugated to a specific adding machine in such a manner that the semi-conjugation 
map is almost everywhere injective with respect to the single ergodic invariant probability measure associated with the adding machine.

Even though we chose to consider the simplest possible sequence of winding matrices from Theorem 3.12, the lemmas, propositions, and proofs within this section can be easily modified to prove the result when considering any sequence of winding matrices that matches the criteria laid out in the statement of Theorem 3.12. This results in the following more general theorem.

Theorem 4.24. Given $m \geq 2$, there exists a logistic unimodal map $q$ with critical point c satisfying that $\omega(c)$ is a non-uniquely ergodic minimal Cantor set possessing $m$ measures. Moreover, this logistic map satisfies that $(\omega(c), q)$ can be semiconjugated to a specific adding machine in such a way that the semi-conjugation map is almost everywhere injective with respect to the single ergodic invariant probability measure associated with the adding machine.

\subsection{Future Work}

In this chapter, we proved that there exist finitely non-uniquely ergodic minimal Cantor sets obtained as the omega-limit set of certain logistic unimodal maps that may be semi-conjugated to a corresponding adding machine in such a way that the semi-conjugation map is almost everywhere injective with respect to the unique ergodic measure on the adding machine. It is likely that the lemmas, propositions, and proofs contained within this chapter can be modified in order to prove the same result where the logistic map is infinitely non-uniquely ergodic instead.

Conjecture 4.25. There exists a logistic unimodal map $q$ with critical point $c$ satisfying that $(\omega(c), q)$ is an infinitely non-uniquely ergodic minimal Cantor that can be semi-conjugated to a specific adding machine in such a way that the semiconjugation map is almost everywhere injective with respect to the single ergodic 
invariant probability measure associated with the adding machine.

\section{List of References}

[1] J. Milnor and W. Thurston, "On iterated maps of the interval," in Dynamical Systems, ser. Lecture Notes in Mathematics, J. Alexander, Ed. Springer Berlin Heidelberg, 1988, vol. 1342, pp. 465-563.

[2] S. Isola and A. Politi, "Universal encoding for unimodal maps," Journal of Statistical Physics, vol. 61, no. 1, pp. 263-291, 1990.

[3] S. Isola, "On a set of numbers arising in the dynamics of unimodal maps," Far East Journal of Dynamical Systems, vol. 6, no. 1, pp. 79-96, 2004.

[4] M. I. Cortez and J. Rivera-Letelier, "Invariant measures of minimal post-critical sets of logistic maps," Israel Journal of Mathematics, vol. 176, no. 1, pp. 157193, 2008. 


\section{BIBLIOGRAPHY}

Alvin, L., "Uniformly recurrent sequences and minimal Cantor omega-limit sets," Fund. Math., vol. 231, pp. 273-284, 2015.

Birkhoff, G., "Quelques théorèmes sur le mouvement des systèmes dynamiques," Bull. Soc. Math. France, vol. 40, pp. 305-323, 1912.

Brin, M. and Stuck, G., Introduction to Dynamical Systems. Cambridge University Press, 2002.

Bruin, H., "Minimal Cantor systems and unimodal maps," Journal of Difference Equations and Applications, vol. 9(3-4), pp. 305-318, 2003.

Cantor, G., "Über unendliche, lineare Punktmannichfaltigkeiten," Math. Ann., vol. 21, pp. 545-591, 1883.

Collas, P. and Klein, D., "An ergodic adding machine on the Cantor set," L'Enseignement Mathmatique, vol. 40, no. 249, 1994.

Cortez, M. I. and Rivera-Letelier, J., "Invariant measures of minimal post-critical sets of logistic maps," Israel Journal of Mathematics, vol. 176, no. 1, pp. 157-193, 2008.

de Melo, W. and van Strien, S., One-Dimensional Dynamics. Springer, 1993.

Devaney, R., An Introduction to Chaotic Dynamical Systems. New York: Hafner Publishing Company, 1989.

Downarowicz, T. and Durand, F., "Factors of toeplitz flows and other almost 1-1 extensions over group rotations," Journal of Difference Equations and Applications, vol. 90, pp. 57-72, 2002.

Fokkink, R. and Oversteegen, L., "Homogeneous weak solenoids," Trans. Amer. Math. Soc., vol. 354(9), pp. 3743-3755, 2002.

Gambaudo, J. and Martens, M., "Algebraic topology for minimal Cantor sets," Annales Henri Poincaré, vol. 7, pp. 423-446, 2006.

Hakobyan, H., "Cantor sets which are minimal for quasi symmetric maps," Journal of Contemporary Mathematical Analysis (Armenian Academy of Sciences), vol. 41(2), pp. 5-13, 2006.

Hohn, F., Elementary Matrix Algebra (3rd edition). MacMillan, 1973.

Isola, S., "On a set of numbers arising in the dynamics of unimodal maps," Far East Journal of Dynamical Systems, vol. 6, no. 1, pp. 79-96, 2004. 
Isola, S. and Politi, A., "Universal encoding for unimodal maps," Journal of Statistical Physics, vol. 61, no. 1, pp. 263-291, 1990.

Knopp, K., Theory and Application of Infinite Series. New York: Hafner Publishing Company, 1947.

Lyubich, M. and Milnor, J., "The Fibonacci unimodal map," Journal of the American Mathematical Society, vol. 6, Number 2, 1993.

Mane, R., Ergodic Theory and Differentiable Dynamics. Springer-Verlag, 1987.

Martens, M., "Distortion results and invariant Cantor sets of unimodal maps," Ergodic Theory and Dynamical Systems, vol. 14, pp. 331-349, 1992.

McCord, M., "Inverse limit sequences with covering maps," Trans. Amer. Math. Soc., vol. 114(30), pp. 197-209, 1965.

Milnor, J. and Thurston, W., "On iterated maps of the interval," in Dynamical Systems, ser. Lecture Notes in Mathematics, Alexander, J., Ed. Springer Berlin Heidelberg, 1988, vol. 1342, pp. 465-563.

West, D., Introduction to Graph Theory: Second Edition. Addison-Wesley Publishing Company,, 2001.

Winckler, B., "Renormalization of Lorenz maps," Ph.D. dissertation, Royal Institute of Techology, Sweden, 2011. 